Aus: BIBLIOTHEK Forschung und Praxis,

Band 28, Heft 1, Seiten 35-59,

ISSN (Print) 0341-4183,

DOI: https://doi.org/10.1515/BFUP.2004.35.

Heike Andermann, Andreas Degkwitz

\title{
Neue Ansätze in der wissenschaftlichen Informationsversorgung ${ }^{1}$
}

\section{Ein Überblick über Initiativen und Unternehmungen auf dem Gebiet des elektronischen Publizierens}
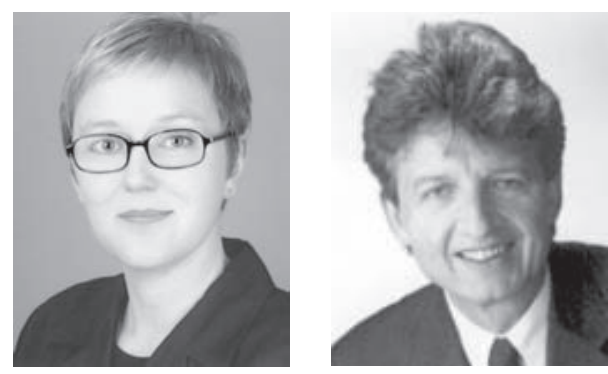

Der Bericht bietet einen Überblick und eine vorläufige Einschätzung der Entwicklungen auf dem Gebiet des elektronischen Publizierens. Es werden Initiativen und Unternehmungen vorgestellt, die sich in die Aktionsfelder „neues Geschäftsmodell“, „Selbstorganisation der Wissenschaften“, „neue Kooperationsformen“ und „Kostensenkung durch Wettbewerb" differenzieren lassen. Darüber hinaus werden neue Modelle für die Begutachtung (peer review) wissenschaftlicher Publikationen beschrieben.

New approaches to scientific information services. An overview about initiatives and enterprises in the field of electronic publishing

The report offers an overview and a preliminary estimate about developments in the field of electronic publishing. It presents initiatives and enterprises, which can be distinguished in four fields of action: „new business model”, „selforganisation of the sciences”, „new forms of cooperation” and "cost reduction through competition”. Additionally new forms of peer review in the process of scientific communication are described

Nouveaux approches aux services d'information scientifiques. Une vue d'ensemble sur les initiatives et les entreprises dans le secteur des publications électroniques

Le rapport donne une vue d'ensemble et une estimation préliminaire du développement dans le domaine de la publiquation électronique. Les différentes initiatives sont présentées dans les champs d'activités „nouveau type d'affaire“, "'organisation autonome de la science“, „nouvelle forme de coopération" et "la réduction des coûts". De plus de nouvelles expertises des publiquations scientiques seront décrit. 
Inhalt

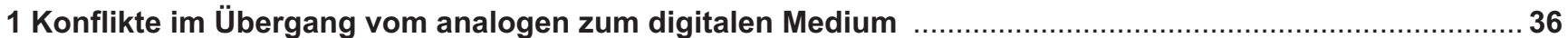

2 Elektronisches Publizieren - Initiativen und Unternehmungen ..................................................... 42

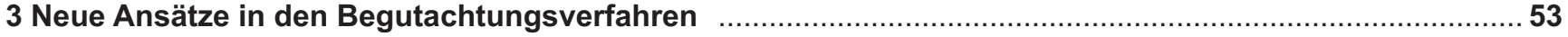

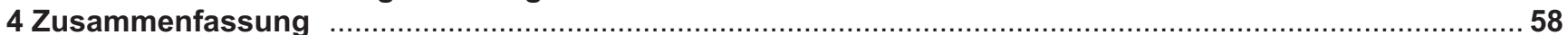

\section{Konflikte im Übergang vom analogen zum di- gitalen Medium}

Im Übergang vom analogen zum digitalen Medium verschärfen sich die Konflikte zwischen den Akteuren in der Wertschöpfungskette wissenschaftlicher Information. Es fehlen wissenschaftsfreundliche ${ }^{2}$ Preisgestaltungen für Fachzeitschriften, mediengerechte Geschäftsmodelle für die digitale Umgebung und adäquate rechtliche Rahmenbedingungen für die Publikation und Verbreitung der wissenschaftlichen Information im digitalen Umfeld.

Mit der Transformation zum digitalen Medium eröffnen sich jedoch neue Wege der wissenschaftlichen Kommunikation und Publikation. Digitalisierung ermöglicht die Vervielfältigung der Dokumente, ohne die Qualität der Originale zu gefährden, und die Internettechnologien machen die rasche globale Verbreitung der wissenschaftlichen Information erstmals möglich ${ }^{3}$. Die beliebige Vermehrbarkeit und die weltweiten Verbreitungsmöglichkeiten stellen die für die Printumgebung entwickelten Exklusivrechte auf die Verwertung und Verbreitung der wissenschaftlichen Information durch die Verlage zunehmend in Frage. Auch erscheinen die existierenden Geschäftsmodelle der Subskription, Lizenzierung und des pay-per-view, die den Zugriff auf die wissenschaftliche Information auf grundlegende Weise einschränken, nicht länger adäquat (Restricted Access).

Der vorliegende Bericht verfolgt das Ziel, einen Überblick über bestehende Initiativen und Unternehmungen zu geben, die durch den Einsatz neuer Produktions- und Verbreitungstechnologien zu einer Neustrukturierung des wissenschaftlichen Publikations- und Kommunikationsprozesses beitragen. Auf diesem Gebiet sind gegenwärtig vier Aktionsfelder erkennbar:

1. die Entwicklung neuer Geschäftsmodelle, die einen kostenlosen Zugang zur wissenschaftlichen Information gewährleisten,

2. der Aufbau wissenschafts- bzw. hochschuleigener Kommunikationsstrukturen und Publikationsumgebungen,

3. die Entwicklung neuer Kooperationsformen für die Produktion und Distribution wissenschaftlicher Information,

4. die Herausgabe von Zeitschriften als direkte Konkurrenzprodukte zu hochpreisigen Zeitschriften kommerzieller Verlage mit dem Ziel, Einfluss auf die Preisentwicklungen der jeweiligen Zeitschrift zu nehmen.

Darüber hinaus zeichnen sich auch Veränderungen in den Begutachtungsverfahren (dem Peer Review) ab, die hier kurz vorgestellt werden.

\subsection{Die Wertschöpfungskette wissenschaftlicher Information}

Fachzeitschriften sind nicht der einzige Ort wissenschaftlicher Kommunikation ${ }^{4}$, in dem gegenwärtigen Wissenschaftssystem nehmen sie jedoch eine herausragende
Stellung ein, wenn es um die Bewertung der Relevanz des publizierten Inhalts und somit um die Reputation der Wissenschaftler geht ${ }^{5}$. Für Berufungs- und Beförderungsprozesse stellen die Veröffentlichungen in Fachzeitschriften mit einem hohen Renommee eine wichtige Entscheidungsgrundlage dar. Im Kontext wissenschaftlicher Kommunikation übernehmen sie mehrere Funktionen:

Registrierung: Erst mit der Veröffentlichung eines Aufsatzes in einer anerkannten Zeitschrift können die Wissenschaftler Anspruch auf die wissenschaftliche Entdeckung und auf die Urheberschaft der gewonnenen Erkenntnisse erheben (Entdeckungspriorität).

Zertifizierung: Mit der Veröffentlichung der Forschungsergebnisse in einer Zeitschrift wird die Arbeit der Wissenschaftler anerkannt und sichtbar gemacht. Auch bei der Rezeption sind die Wissenschaftler (schon aus zeitlichen Gründen) auf den Qualitätsfilter, den die Fachzeitschriften (und ihre zertifizierten Artikel) darstellen, angewiesen.

Verbreitung/Sichtbarkeit: Die Maximierung der Sichtbarkeit ist das wesentliche Ziel der Veröffentlichung. Mit der Publikation in einer Fachzeitschrift wollen die Wissenschaftler die für sie relevante wissenschaftliche Gemeinschaft erreichen.

Archivierung: Aufbewahrung und Verfügbarmachung der wissenschaftlichen Inhalte für nachfolgende Generationen ${ }^{6}$.

1 Der Bericht ist im Rahmen des DFG-Projekts „Perspektiven für den Bezug elektronischer Fachinformation in der Bundesrepublik Deutschland" entstanden. Nähere Informationen zu dem Projekt finden sich unter <http://www. epublications.de>.

${ }_{2}^{2}$ Als wissenschaftsfreundlich wird eine größtmögliche Verfügbarkeit wissenschaftlicher Information zu einem geringstmöglichen Preis verstanden.

${ }^{3}$ Vgl. Kuhlen, Rainer (2002): Medienprodukte im Netz. Zwischen Kommerzialisierung und freiem Zugang. Überarbeitete Version eines Vortrags auf der Tagung „Digital Rights Management" am 20.11.2002. Zugang: <http://www.inf-wiss. uni-konstanz.de/People/RKNortaege02-Web/abstract-vortragmuenchen-rk-201102.pdf> (15.02.03).

${ }^{4}$ Vgl. hierzu Odlyzko, Andrew: The future of scientific communication. In: Wouters, Paul; Schroede, Peter (Hrsgg.): Access to publicity Financed Research. The Global Research Village III. Amsterdam 2000. S. 273-278. Zugang: <http:// www.dtc.umn.edu/ odlyzko/doc/future.scientific.comm.pdf> (15.02.03).

${ }^{5}$ Die männliche Schreibweise wird in diesem Bericht wegen der Lesbarkeit verwendet. Selbstverständlich sind hiermit auch Wissenschaftlerinnen, Autorinnen etc. gemeint.

${ }^{6}$ Vgl. Crow, Raym: Scholarly Publishing and Academic Resources Coalition (SPARC): The Case for Institutional Repositories. A SPARC Position Paper. 2002. S. 7 f. Zugang: <http:// www.arteorig/sparic/home//indexdaspi?page 
Für den Publikationsprozess im analogen Umfeld („Printwelt") hat sich eine Wertschöpfungskette herausgebildet, in der die Zusammenarbeit zwischen Urheber (Wissenschaftler), Verwerter (Verlage) und Abnehmer (Bibliotheken) der wissenschaftlichen Information durch entsprechende Geschäftsmodelle und rechtliche Rahmenbedingungen geregelt ist. Hierbei nehmen die Verlage eine zentrale Rolle bei der Produktion und Verbreitung wissenschaftlicher Information ein ${ }^{7}$. Die Wertschöpfungskette wissenschaftlicher Information in der Printumgebung hat folgende Struktur: deren Bibliotheken - im Mittelpunkt, die für Forschende, Lehrende und Studierende die wissenschaftliche Information erwerben, die diese wiederum zu Forschungs-, Lehr- und Studienzwecken einsetzen.

Die folgende Tabelle verdeutlicht nochmals die Aufgabenverteilung der Akteure in der Wertschöpfungskette ${ }^{13}$ :

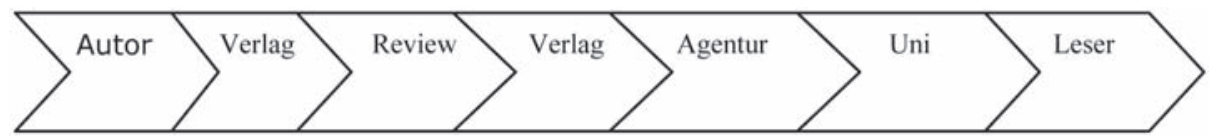

Graphik 1: Wertschöpfungskette wissenschaftlicher Information im analogen Kontext

Die Autoren - Urheber der wissenschaftlichen Information - stehen am Beginn des Wertschöpfungsprozesses. Ihr Interesse ist die Veröffentlichung von wissenschaftlichen Ergebnissen in Zeitschriften mit einem hohen Renommee und großer Reichweite. Nur durch die Publikation und die damit erzielte Aufmerksamkeit bzw. Sichtbarkeit besteht die Möglichkeit, die notwendige Reputation aufzubauen. Je renommierter die Zeitschrift ist, indem der Artikel publiziert wird, desto größer sind die Chancen auf Anerkennung ${ }^{8}$. Die Interessen der Verlage sind anderer Natur. Verlage benötigen in der Regel lange Zeiträume, um Zeitschriften als „Markenartikel“, d.h. mit einem hohen Renommee und einer großen Reichweite, in der Wissenschaftlergemeinschaft zu etablieren. Gelingt dies, ist die Markteintrittsbarriere für konkurrierende Zeitschriften sehr hoch. Meier beschreibt dies für den STM-Zeitschriftenmarkt folgendermaßen: „Wird ein signifikanter Marktanteil und entsprechendes Renommee auf dem STM-Zeitschriftenmarkt erreicht, ist der Markteintritt für Konkurrenzverlage erschwert. Einem neuen, konkurrierenden Journal bleiben dann nur die Artikel mit geringerem qualitativen Anspruch, so lange der Markt nicht zwei Top-Journale mit hoher Qualität zulässt. [...] Das Risiko eines bereits etablierten Journals, durch ein später auf dem Markt kommenden Konkurrenzprodukt verdrängt zu werden, ist daher sehr gering." ${ }^{9}$

Unterstützt werden die Verlage durch die für die Printumgebung entwickelten rechtlichen Rahmenbedingungen, die den Verlagen das exklusive Recht für die Verbreitung und Verwertung des Artikels zusichern. Die Verlagspolitik besteht darin, nur solche Artikel zu veröffentlichen, die in einer anderen Zeitschrift noch nicht erschienen sind ${ }^{10}$. Verbreitet sind sogenannte „Buy-out-Verträge“. Charakteristisch für diesen Vertragstyp ist, dass der Urheber sämtliche Nutzungsarten (sachlich, zeitlich, räumlich) gegen eine einmalige Zahlung an den Werkverwerter überträgt. Für den Verwerter der wissenschaftlichen Arbeit liegt der Vorteil darin, dass sich die Übertragung auf alle Medienarten bezieht ${ }^{11}$. „Damit verschafft sich der Verwerter die Option [...], die Verwertung nicht nur in der gedruckten Zeitschrift zu besorgen, sondern auch auf Diskette, CDROM oder künftig auf DVD sowie online vergütungsfrei oder vergütungspflichtig in einem pay per view-Verfahren im Internet." 12

Als Abnehmer der wissenschaftlichen Information stehen hier die institutionellen Abnehmer - die Universitäten bzw.
${ }^{7}$ Vgl. Roosendaal, Hans E.: Market Transformation? Changes in the value chain. 2001. Zugang: <http://www.niwi.knaw. $\mathrm{nl} / \mathrm{ccsc} /$ talks/roosendaal.ppt> (23.07.03). Vgl. auch Kuhlen, Rainer: New Code - New Law. Oder doch eher neue Geschäftsmodelle für urheberrechtsrelevante elektronische Güter? (2002). Zugang: <http://www.inf-wiss.uni-konstanz. de/People/RK/Vortraege02-web/vortrag-st-gallen-291002. pdf> (23.02.03).

8 Vgl. hierzu ausführlicher Meier, Michael: Returning Science to the Scientists. Der Umbruch im STM-Zeitschriftenmarkt unter Einfluß des Electronic Publishing. München 2002. S. $19 \mathrm{ff}$.

${ }^{9}$ Meier (Anm. 8) S. 89.

10 Diese Vorgehensweise wird auch als "Ingelfinger-Gesetz" bezeichnet, nach dem Herausgeber der Zeitschrift New England Journal of Medicine Franz Ingelfinger. Von Stevan Harnad werden diese folgendermaßen zusammengefasst: „(1) Public Health must be protected: only refereed research, reviewed and certified by the qualified specialists, should be made public. (2) The refereeing and certification system must be protected. Referees are a scarce resource, donating their valuable time for free. There is no justification for squandering their time on a paper that has already been published without certification, or one that has already been certified and published by another journal. (3) The journal's (and author's) priority and prestige must be protected: readers will not read or cite a journal whose contents have already appeared elsewhere. (4) The journal's revenue streams must be protected: subscribers will not subscribe to a journal whose contents have already appeared elsewhere. Without that revenue, the research cannot be refereed or published at all." In: Harnad, Stevan: Ingelfinger Over-Ruled. The Role of the Web in the Future of Refereed Medical Journal Publishing. Preprint. (2000) Zugang: <http://www.ecs.soton.ac.uk/ harnad/Papers/Harnad/ harnad00.lancet.htm> (20.02.03).

11 Vgl. hierzu ausführlicher Schröter, Madeleine: Der (Copyright-)Vertrag des Wissenschaftlers mit dem Verlag. In: Forschungszentrum Jülich (Hrsg.): Die Zukunft des wissenschaftlichen Publizierens. Der Wissenschaftler im Dialog mit Verlag und Bibliothek. Schriftenreihe des Forschungszentrums Jülich, Bd.10. Jülich 2002. S. 42 ff.

12 Ebd., S. 42.

${ }^{13}$ Vgl. hierzu Crow (Anm. 6) S. 9. Zugang: <http://www.arl.

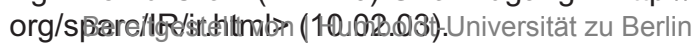




\begin{tabular}{|c|c|c|c|}
\hline Funktion & Prozess & Akteur & $\begin{array}{c}\text { Prozess- } \\
\text { beauftragter }\end{array}$ \\
\hline Registrierung & $\begin{array}{l}\text { Einreichen des Papiers bei } \\
\text { der Zeitschrift / dem Verlag }\end{array}$ & $\begin{array}{l}\text { Akademischer Autor/ } \\
\text { Wissenschaftler }\end{array}$ & Verlag \\
\hline Zertifizierung & Peer Review & Akademische Reviewer & Verlag \\
\hline $\begin{array}{c}\text { Verbreitung/ } \\
\text { Zugänglichkeit }\end{array}$ & $\begin{array}{c}\text { Verbreitung } \\
\text { und } \\
\text { Auswahl der Zeitschrift }\end{array}$ & $\begin{array}{c}\text { Verlage } \\
\text { Bibliotheken }\end{array}$ & $\begin{array}{c}\text { Verlage } \\
\text { Akademische Institution/ } \\
\text { Bibliothek }\end{array}$ \\
\hline Archivierung & dauerhafter Zugriff & Bibliotheken & $\begin{array}{c}\text { Akademische Institution/ } \\
\text { Bibliothek }\end{array}$ \\
\hline
\end{tabular}

Tabelle 2: Akteure/Prozesse in der traditionellen Wertschöpfungskette

In dem für die Printumgebung entwickelten Organisationsmodell treten die Hochschulen nur als Abnehmer, nicht als Produzenten und Distributoren wissenschaftlicher Information auf.

Die Balance zwischen den Akteuren der Wertschöpfungskette wird jedoch durch die seit Jahren andauernde aggressive Preispolitik großer kommerzieller Verlage nachhaltig gestört. Preissteigerungen und Konzentrationsprozesse auf dem Verlagsmarkt machen die Suche nach neuen Perspektiven für die Produktion, Distribution und Archivierung wissenschaftlicher Information dringend erforderlich.

\subsection{Preissteigerungen für die wissenschaftliche In- formation}

Für die Auswirkungen der Preissteigerungen wissenschaftlicher Zeitschriften auf die wissenschaftliche Informationsversorgung hat sich seit einigen Jahren der Begriff der „Krise der wissenschaftlichen Informationsversorgung" durchgesetzt. Gemeint ist die wachsende Lücke zwischen den Preisen für die wissenschaftliche Information und deren Finanzierbarkeit durch die Bibliotheken mit der Folge, dass Bibliotheken nicht nur Zeitschriften abbestellen, sondern auch die Mittel für den Monographienerwerb in nicht-naturwissenschaftlichen Disziplinen reduziert werden müssen.

In den vergangenen Jahren sind die Kosten für wissenschaftliche Zeitschriften insbesondere in den natur-, ingenieurswissenschaftlichen und medizinischen Fachgebieten (STM) stark angestiegen. Die Bundesvereinigung deutscher Bibliotheksverbände weist darauf hin, dass in einem Zeitraum von nur vier Jahren (1997-2000) eine Preissteigerung von über $50 \%$ in diesen Fachgebieten zu verzeichnen ist. Insbesondere bei den Kernzeitschriften ist eine überproportionale Preissteigerung erkennbar. In einer repräsentativen Auswahl von 20 STM-Zeitschriften ${ }^{14}$ lässt sich eine Preissteigerung von über $200 \%$ bei mehr als $50 \%$ (11 Zeitschriften) der Zeitschriften feststellen, bei 5 Zeitschriften ist eine Preissteigerung von $150 \%$ und bei den verbleibenden 4 Zeitschriften eine Preissteigerung von über $100 \%$ zu beobachten ${ }^{15}$. Der für die Länder Deutschland, Österreich und Schweiz herausgegebene HarrassowitzPreisindex zeigt für diese Fachgebiete in den Jahren von 1996-2002 eine Preissteigerung von etwa $50 \%$ an ${ }^{16}$. In den USA ermittelt die American Library Association die Preissteigerungen mit Hilfe des U.S. Periodical Price Index. In einem Zeitraum von 10 Jahren (1991-2000) ist für die verschiedenen Fächer eine durchschnittliche jährliche Preissteigerung in folgender Höhe festzustellen ${ }^{17}$ (vgl. Tabelle 3):
Eine Betrachtung der Preisentwicklungen auf der Ebene durchschnittlicher Preissteigerungen pro Fachgebiet ist jedoch nur begrenzt geeignet, die Situation adäquat einzuschätzen. Preissteigerungen finden sich insbesondere bei solchen Zeitschriften, die über ein hohes Renommee innerhalb der wissenschaftlichen Gemeinschaft verfügen und aus diesem Grund als Kernzeitschrift des jeweiligen Fachgebietes gehalten werden müssen (,need-to-knowProdukt").

Die folgende Tabelle, herausgegeben vom Verband amerikanischer wissenschaftlicher Bibliotheken (Association of Research Libraries/ARL), gibt einen Überblick über die Preisentwicklungen wissenschaftlicher Zeitschriften für die Jahre 1995 und 2001 für ausgewählte Kernzeitschriften in den STM-Gebieten ${ }^{18}$. Beobachtungen auf der Ebene einer einzelnen Zeitschrift vermitteln einen besseren Eindruck der Preisentwicklungen (vgl. Graphik 4).

Exemplarisch für die europäische Situation lässt sich die Preisentwicklung am Beispiel des Landes Großbritannien darstellen ${ }^{19}$. Die von SCONUL (Standing Conference of National and University Libraries) und der Loughborough University herausgegebene Graphik umfasst einen Betrach-

${ }^{14}$ Betrachtungszeitraum: 1992-1999.

15 Vgl. Griebel, Rolf; Tscharntke, Ulrike: Analyse der Etatsituation wissenschaftlicher Bibliotheken 1998/1999. München 1999. S. 12.

${ }^{16}$ Griebel, Tscharntke (Anm. 15).

17 Albee, Barbara; Dingley, Brenda: U.S. Periodical Prices2000. In: American Libraries (May 2000) 2000, S. 78-86. Zugang: <http://archive.ala.org/alonline/archive/periodicals 00.pdf > (10.03.03) (auf Dollarbasis und ohne Berücksichtigung russischer Übersetzungen).

${ }_{18}$ Vgl. hierzu Case, Mary M.: „Scholarly Communication. A system in crisis." (2001). Zugang: <http://www.lib.ohio-state. edu/Staff/scholcom/case925.html> (10.02.03).

19 Im STM-Zeitschriftenmarkt ist seit den siebziger Jahren eine fortdauernde Kostenerhöhung zu beobachten, die von den Verlagen folgendermaßen begründet wird: sinkende Privatabonnements machen eine Erhöhung der Preise für institutionelle Kunden (Subskriptions-Anpassungen) erforderlich, Investitionen in elektronische Publikationssysteme, Parallelbezug von Print- und elektronischen Zeitschriften verursachen Mehrkosten, die an die institutionellen Abnehmer weitergegeben werden. Vgl. Meier (Anm. 7) S. 31 und S. 99 fí.ereitgestellt von | Humboldt-Universität zu Berlin 


\begin{tabular}{|c|c|c|}
\hline Fachgebiete & $\begin{array}{c}\text { durchschnittliche Preissteigerungen/ } \\
\text { Fachgebiet/Jahr }\end{array}$ & $\begin{array}{l}\text { durchschnittliche Preissteigerungen/ } \\
\text { Jahr für die Fachgebiete der: }\end{array}$ \\
\hline General Interest Periodicals & 3,6 & \multirow{5}{*}{$\begin{array}{c}\text { Geisteswissenschaften } \\
44,9\end{array}$} \\
\hline Children`s Periodicals & 3,7 & \\
\hline Fine and Applied Arts & 4,4 & \\
\hline Literature and Language & 6,2 & \\
\hline Philosophy and Religion & 6,7 & \\
\hline Business and Economics & 8,5 & \multirow{11}{*}{$\begin{array}{l}\text { Sozial- } \\
\text { und Wirtschaftswissenschaften } \\
7,6\end{array}$} \\
\hline Education & 8,3 & \\
\hline History & 6,0 & \\
\hline Journalism and Communications & 6,7 & \\
\hline Labor and Industrial Relations & 8,1 & \\
\hline Law & 6,5 & \\
\hline Library and Information Sciences & 5,3 & \\
\hline Physical Education and Recreation & 4,9 & \\
\hline Political Science & 9,4 & \\
\hline Psychology & 9,8 & \\
\hline Sociology and Anthropology & 9,0 & \\
\hline Agriculture & 8,2 & \multirow{8}{*}{$\begin{array}{c}\text { Naturwissenschaften, Medizin } \\
\text { und } \\
\text { Technik } \\
9,8\end{array}$} \\
\hline Chemistry and Physics & 12,2 & \\
\hline Engineering & 10,5 & \\
\hline Home Economics & 6,1 & \\
\hline Industrial Arts & 7,4 & \\
\hline Mathematics, etc. & 10,7 & \\
\hline Medicine & 11,8 & \\
\hline Zoology & 11,8 & \\
\hline
\end{tabular}

Tabelle 3: U.S.Periodical Price Index 1991-2000

\section{SAMPLE OF JOURNAL PRICES}

$\begin{array}{lccc} & \mathbf{1 9 9 5} & \mathbf{2 0 0 1} & \text { \% Change } \\ \text { Brain Research } & \$ 10,181 & \$ 17,444 & 71.3 \% \\ \text { Biochim. Biophys. Acta } & \$ 7,555 & \$ 12,127 & 60.5 \% \\ \text { Chem. Phys. Letters } & \$ 5,279 & \$ 9,637 & 82.6 \% \\ \text { Tetrahedron Letters } & \$ 5,119 & \$ 9,036 & 76.5 \% \\ \text { Eur. Jrnl. of Pharmacology } & \$ 4,576 & \$ 7,889 & 72.4 \% \\ \text { Gene } & \$ 3,924 & \$ 7,443 & 89.7 \% \\ \text { Inorganica Chim. Acta } & \$ 3,611 & \$ 6,726 & 86.3 \% \\ \text { Intl. Jrnl. of Pharmaceutics } & \$ 3,006 & \$ 5,965 & 98.4 \% \\ \text { Neuroscience } & \$ 3,487 & \$ 6,270 & 79.8 \% \\ \text { Theoretical Computer Science } & \$ 2,774 & \$ 4,608 & 66.1 \% \\ \text { Jrnl. of Exp. Marine Bio. \& Eco. } & \$ 1,947 & \$ 3,501 & 79.8 \%\end{array}$

Graphik 4: Preissteigerungen ausgewählter STM-Zeitschriften

tungszeitraum von 10 Jahren (1991-2000). Die Graphik verdeutlicht, dass die Preissteigerungen für die wissenschaftlichen Zeitschriften nicht nur zu einer rückläufigen
Anzahl der Zeitschriftenabonnements in den wissenschaftlichen Bibliotheken führt, sondern auch zu einem rückläufigen Bestandsaufbau|imaMlenographieabereioin. 
THE EFFECT OF JOURNAL PRICES ON UK ACADEMIC LIBRARIES

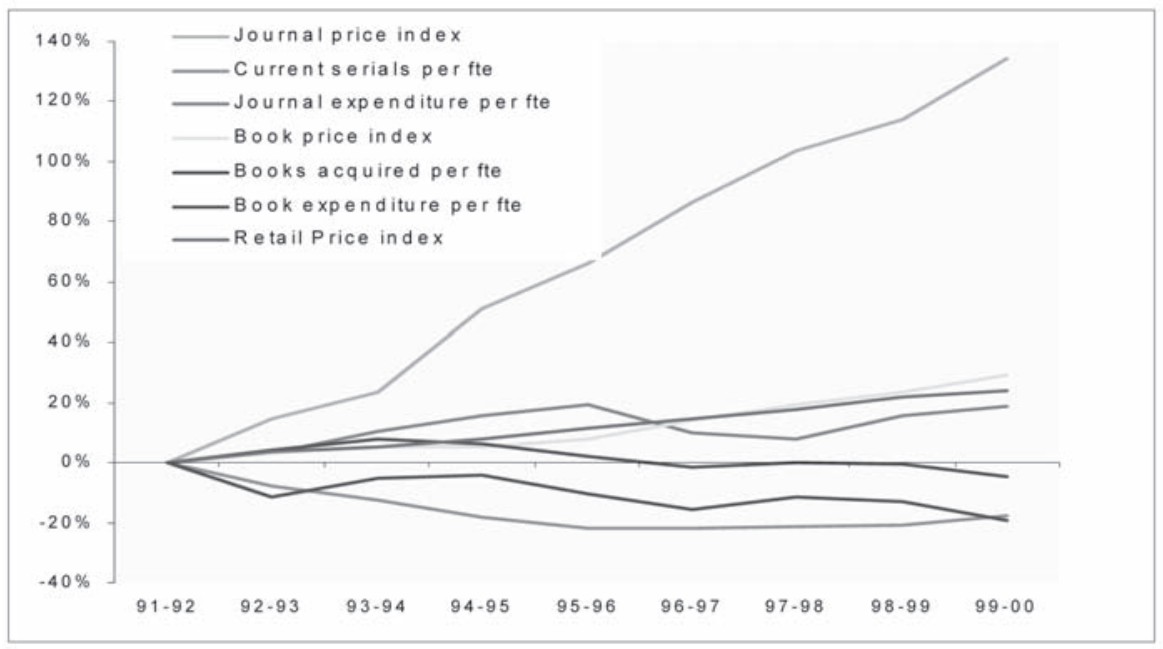

Graphik 5: Auswirkungen der Preissteigerungen auf die Erwerbung

Von bibliothekarischer Seite wird bereits seit mehreren Jahren auf die Folgen der zunehmenden Preissteigerungen hingewiesen. 1999 hat die Erwerbungskommission in einem offenen Brief an die Verlage deren Preispolitik kritisiert ${ }^{20}$. Im Jahr 2001 hat auch der Deutsche Bibliotheksverband in seiner Stellungnahme zum Strategiekonzept „Zukunft der wissenschaftlichen und technischen Information" auf die krisenhafte Entwicklung als Folge zunehmender Konzentrationsprozesse und einer monopolistischen Preispolitik im Verlagswesen hingewiesen ${ }^{21}$. Die Wirkung dieser Statements ist angesichts der weiter ansteigenden Preise für wissenschaftliche Information eher gering.

\subsection{Struktur des Verlagsmarktes}

Große kommerzielle Verlage dominieren den Markt wissenschaftlicher Information. Die in den vergangenen Jahren zu beobachtenden Verlagsfusionen tragen weiter dazu bei, das Portfolio einiger Verlage zu vergrößern. Im Jahr 1998 ergibt sich für die in den Indices des Institute of Scientific Information (ISI) nachgewiesenen Zeitschriften folgende Verteilung auf die Verlage ${ }^{22}$ :

\begin{tabular}{|l|c|c|}
\hline \multicolumn{1}{|c|}{ Publisher } & Type of Publisher & $\begin{array}{c}\text { Number of } \\
\text { ISI-Rated } \\
\text { Journals 1998 }\end{array}$ \\
\hline Elsevier Science & Commercial & 1347 \\
\hline Wolters-Kluwer & Commercial & 552 \\
\hline $\begin{array}{l}\text { Blackwell } \\
\text { Publishers }\end{array}$ & Commercial & 341 \\
\hline Bertelsmann & Commercial & 326 \\
\hline Wiley & Commercial & 279 \\
\hline Taylor \& Francis & Commercial & 275 \\
\hline Sage & Commercial & 123 \\
\hline Karger & Commercial & 101 \\
\hline $\begin{array}{l}\text { Institute of } \\
\text { Electrical and } \\
\text { Electronics } \\
\text { Engineers }\end{array}$ & Society & 93 \\
\hline
\end{tabular}

\begin{tabular}{|l|c|c|}
\hline $\begin{array}{l}\text { Cambridge } \\
\text { University Press }\end{array}$ & University Press & 84 \\
\hline $\begin{array}{l}\text { Oxford University } \\
\text { Press }\end{array}$ & University Press & 83 \\
\hline Marcel Dekker & Commercial & 76 \\
\hline Holtzbrinck & Commercial & 67 \\
\hline $\begin{array}{l}\text { America Institute } \\
\text { of Physics }\end{array}$ & Society & 41 \\
\hline $\begin{array}{l}\text { Scandinavian } \\
\text { University Press }\end{array}$ & University Press & 39 \\
\hline $\begin{array}{l}\text { University of } \\
\text { Chicago Press }\end{array}$ & University Press & 39 \\
\hline $\begin{array}{l}\text { Mary Ann Liebert } \\
\text { Inc. }\end{array}$ & Commercial & 34 \\
\hline $\begin{array}{l}\text { IOP Publishing } \\
\text { Ltd. (Institute of } \\
\text { Physics) }\end{array}$ & Society & 32 \\
\hline $\begin{array}{l}\text { American } \\
\text { Chemical Society }\end{array}$ & Society & 31 \\
\hline Havas & 31 \\
\hline $\begin{array}{l}\text { Others (2.028 } \\
\text { Publishers) }\end{array}$ & & 3716 \\
\hline Grand Total & & $\mathbf{7 7 1 0}$ \\
\hline
\end{tabular}

Tabelle 6: Anzahl der im ISI nachgewiesenen Zeitschriften

20 Zeitschriftenpreise 1999 - Offener Brief. In: Bibliotheksdienst 33 (1999) S. 311-313.

21 Deutscher Bibliotheksverband: Stellungnahme des Deutschen Bibliotheksverbands zum Strategiekonzept „Zukunft der wissenschaftlichen und technischen Information“, 2001. Zugang: <http://www.bibliotheksverband.de/dbv/aktuelles/ adl4-Geisselmann-30-7-011.doc> (14.02.03).

${ }^{22} \mathrm{Vgl}$. Morgan Stanley: Scientific Publishing: Knowledge is Power. Equity Research Europe (2002). Zugang: <http:// www.econ.ucsb.edu/ tedb/Journals/morganstanley.pdf> (23.05:Ba)itgestellt von | Humboldt-Universität zu Berlin 
Der kommerzielle Verlag Reed-Elsevier hat eine überaus dominante Position auf dem Verlagsmarkt. Dieser Rechteverwerter verfügt im Jahr 1998 allein über 17 \% der im ISI nachgewiesenen Zeitschriften. Gemeinsam haben die großen kommerziellen Verlage einen Marktanteil von $50 \%$. Hinsichtlich der Größe ihres Zeitschriftenportfolios haben Fachgesellschaften und Universitätsverlage im internationalen Vergleich einen vergleichsweise geringen Stellenwert.

Bei einer Betrachtung der Erträge und Marktanteile für das Jahr 2001 ist eine Verteilung auf folgende Marktteilnehmer zu beobachten ${ }^{23}$ :

\begin{tabular}{|l|c|c|}
\hline & $\begin{array}{c}\mathbf{2 0 0 1} \\
\text { Revenues } \\
\text { (US\$ mn) }\end{array}$ & $\begin{array}{c}\mathbf{2 0 0 1} \\
\text { Market share } \\
\text { (\%) }\end{array}$ \\
\hline $\begin{array}{l}\text { Reed Elsevier (Elsevier } \\
\text { Science) }\end{array}$ & 1055,3 & 23,3 \\
\hline $\begin{array}{l}\text { American Che mical } \\
\text { Society }\end{array}$ & 357,3 & 7,9 \\
\hline Thomson & 259,0 & 5,7 \\
\hline John Wiley \& Sons & 243,6 & 5,4 \\
\hline $\begin{array}{l}\text { Institute of Electrical \& } \\
\text { Electronics Engineers }\end{array}$ & 200,3 & 4,4 \\
\hline Wolters-Kluwer & 169,3 & 3,7 \\
\hline McGraw-Hill & 146,2 & 3,2 \\
\hline Taylor \& Francis & 144,6 & 3,2 \\
\hline Springer-Verlag & 44,0 & 1,0 \\
\hline Others & 1916,9 & 42,3 \\
\hline Total Scientific Market & $\mathbf{4 5 3 6 , 4}$ & $\mathbf{1 0 0 , 0 \%}$ \\
\hline
\end{tabular}

Tabelle 7: Ertrag und Marktanteil der Verlage

Nicht nur die Größe des Portfolios, sondern auch die Anzahl der Kernzeitschriften in dem Portfolio entscheidet über die Ertragssituation des einzelnen Verlages. Obwohl die Fachgesellschaft American Chemical Society (ACS) lediglich über ein Portfolio von 31 Fachzeitschriften verfügt (Stand 1998), hat sie einen Marktanteil von 7,9\%.

Die Konzentrationsprozesse auf dem Verlagsmarkt sind von Mark McCabe, einem amerikanischen Wirtschaftswissenschaftler, näher untersucht worden ${ }^{24}$. Sein Interesse zielt auf die Analyse der ökonomischen Zusammenhänge zwischen Verlagsfusionen und Preissteigerungen für wissenschaftliche Zeitschriften. McCabe kommt zu dem Ergebnis, dass Verlagsfusionen die Möglichkeiten einer Durchsetzung von Preissteigerungen, insbesondere bei den Kernzeitschriften deutlich erhöhen. Ausgangspunkt seiner Überlegungen ist die Annahme, dass sich die Nachfrage nach wissenschaftlichen Zeitschriften durch die Bibliotheken vom Nachfrageverhalten in anderen Märkten dadurch unterscheidet, dass sich Bibliotheken nicht für ein Produkt mit dem besten Preis-Leistungsverhältnis entscheiden, sondern ihr Angebot an einer heterogenen Kundengruppe ausrichten. Auf der Grundlage der Kosten und des Nutzens fragen sie eine Vielzahl von Zeitschriften des jeweiligen Fachgebietes bzw. verschiedener Fachgebiete nach. Das Ergebnis dieser Nachfrage ist ein Portfolio von Titeln, bei denen die Kriterien Kosten und Nutzung in einem ausgewogenen Verhältnis stehen sollen. Die Ver- lage reagieren auf diesen Portfoliobedarf, indem sie ihre Produktpalette in zwei Gruppen differenzieren:

- thematisch breit angelegte und stark nachgefragte Titel,

- thematisch enge Nischentitel für eine spezialisierte wissenschaftliche Gemeinschaft.

Bei den thematisch engen Nischentiteln handelt es sich in der Regel um Zeitschriften mit hohem Renommee, mit einer loyalen Leserschaft und einem in der Wissenschaftlergemeinschaft anerkannten Herausgebergremium: Faktoren, die eine hohe Einstiegsbarriere für Konkurrenzprodukte bilden mit der Folge, dass diese Zeitschriften quasi konkurrenzlos sind (monopolistische Preissituation). Genau diese Zeitschriften sind sogenannte „need-to-knowProdukte". Sie werden von den Wissenschaftlern stärker nachgefragt und müssen daher von den spezialisierten Bibliotheken abgenommen werden. Aus diesem Grund sind sie gegenüber Preissteigerungen relativ unempfind$\mathrm{lich}^{25}$. Durch die Fusion kommen die Verlage in den Besitz eines Portfolios von Zeitschriften, unter denen sich Fachzeitschriften mit einem hohen Renommee befinden. Preissteigerungen sind durch den Konkurrenzausschluss leichter durchsetzbar. McCabe stellt weiterhin fest, dass es für die Verlage profitabler ist, die Nischentitel zu einem höheren Preis an weniger (spezialisierte) Bibliotheken zu verkaufen, als durch eine Preissenkung die Anzahl der Abnehmer zu erhöhen.

\subsection{Geschäftsmodelle im Übergang zum elektroni- schen Medium}

In der gegenwärtigen Transformationsphase ist die Parallelität von Kaufprinzip für das Printmedium und Lizenzierungsprinzip für die elektronischen Medien zu beobachten. Die Festlegung des Preises für die Fachzeitschriften erfolgt noch in den meisten Fällen auf der Basis des vorhandenen Printbestandes der Bibliothek bzw. des Konsortiums zuzüglich eines Aufschlages für die Lizenz der elektronischen Kopie. In Deutschland wird die Abbestellung des analogen Mediums durch enge Abbestellklauseln, die häufig $5 \%-10 \%$ des Gesamtbestandes nicht überschreiten dürfen, verhindert ${ }^{26}$. Darüber hinaus beziehen Bibliotheken nicht länger einzelne Exemplare einer

${ }^{23}$ Morgan Stanley (Anm. 22) S. 2.

${ }^{24}$ McCabe, Mark: The impact of Publisher Mergers on Journal Prices. An Update. (2001). Zugang: <http://www.arl. org/newsltr/207/jmlprices.html> (25.03.03). McCabe, Mark: Academic Journal Pricing and Market Power. A Portfolio Approach. (2000). Zugang: <http://www.prism.gatech.edu/ $\sim$ mm284/JournPub.PDF> (25.03.03).

${ }^{25}$ Produkte mit fehlender Elastizität zeichnen sich dadurch aus, dass die Nachfrage trotz Preissteigerungen nicht nachlässt. Vgl. ausführlicher zu Preisbildungsstrategien und Preisdifferenzierung Meier (Anm. 8) S. 95 f.

${ }^{26} \mathrm{Vgl}$. hierzu die Ergebnisse einer Befragung deutscher Konsortien, in: Degkwitz, Andreas; Andermann, Heike: Angebots-, Nutzungs- und Bezugsstrukturen elektronischer Fachinformation in Deutschland. In: ABI-Technik Heft 2, S. 122-141.

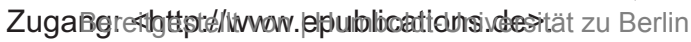


Zeitschrift, sondern Titelpakete, die einen unterschiedlichen Umfang an Zeitschriftentiteln des jeweiligen Verlages umfassen. Bibliotheken vergrößern dadurch das Angebot an wissenschaftlicher Information, jedoch ohne eine Kaufentscheidung nach Kosten-Nutzen-Kriterien treffen zu können.

Bei den großen kommerziellen Verlagen sind derzeit keine Geschäftsmodelle erkennbar, die dem Innovationspotenzial der Internettechnologien besser gerecht werden. Die mit den neuen Technologien einhergehende Globalisierung der Zugriffsmärkte und die beliebige Vermehrbarkeit der digitalen Dokumente machen jedoch die Entwicklung adäquater Geschäftsmodelle zwingend erforderlich. Von einer Übertragung der für die Printumgebung entwickelten Geschäftsmodelle in die digitale Umgebung ist insofern abzuraten, da die möglichen Einsparpotenziale durch die Absenkung der Transaktionskosten nicht an die institutionellen Abnehmer weitergegeben werden ${ }^{27}$. Die Entwicklung genuiner Geschäftsmodelle für die Produktion und Verbreitung elektronischer Dokumenten ist jedoch eine zentrale Aufgabe für die Entwicklung eines Informationsfriedens zwischen den beteiligten Akteuren der Wertschöpfungskette ${ }^{28}$.

2

\section{Elektronisches Publizieren - Initiativen und Un- ternehmungen}

Elektronisches Publizieren ermöglicht nicht nur Einsparmöglichkeiten für die Produktion und Verbreitung wissenschaftlicher Information. Eine Betrachtung des Themas unter ausschließlich monetären Gesichtspunkten wird den Potenzialen, die sich mit den neuen Technologien verbinden, nicht gerecht. Die Internettechnologien eröffnen neue Wege der Produktion, Distribution und Archivierung wissenschaftlicher Information ${ }^{29}$. Sie tragen zu einer Flexibilisierung der Kommunikationsmöglichkeiten innerhalb der Wissenschaftlergemeinschaft bei und beschleunigen diesen Prozess. Die Kommunikation zwischen Wissenschaftlern und die Verbreitung der wissenschaftlichen Information erfolgt weltweit. Mit der globalen Verbreitung der wissenschaftlichen Information durch das Internet wird darüber hinaus eine zentrale Zielsetzung wissenschaftlichen Publizierens realisiert: die Maximierung der Sichtbarkeit für die wissenschaftlichen Arbeiten.

Einhergehend mit der Möglichkeit der globalen Verbreitung des Wissens wird die Forderung nach einem uneingeschränkten Zugang zur wissenschaftlichen Information von der Budapest Open Access Initiative (BOAI) formuliert. Open access im Sinne der BOAI meint, dass Literatur „[...] kostenfrei und öffentlich im Internet zugänglich sein sollte, so dass Interessierte die Volltexte lesen, herunterladen, kopieren, verteilen, drucken, in ihnen suchen, auf sie verweisen und sie auch sonst auf jede denkbare legale Weise benutzen können, ohne finanzielle, gesetzliche oder technische Barrieren jenseits von denen, die mit dem Internet-Zugang selbst verbunden sind"30.

Gegenwärtig lassen sich auf dem Gebiet des elektronischen Publizierens verschiedene Unternehmungen und Initiativen beobachten, die den freien Zugang zur wissenschaftlichen Information auf der Basis eines neuen Geschäftsmodells realisieren.

\subsection{Freier Zugang zur wissenschaftlichen Informa- tion auf der Basis eines neuen Geschäftsmo- dells - Beispiele}

Kennzeichnend für das neue Geschäftsmodell im digitalen Kontext ist der freie, d.h. kostenlose Zugang zur wissenschaftlichen Information nach den Prinzipien der Budapest Open Access Initiative. Die Finanzierung der Produktion und Verbreitung der kostenlos zugänglichen Zeitschriften erfolgt nicht länger durch den Abnehmer, sondern durch den Urheber der wissenschaftlichen Information bzw. durch die Institution, der die Wissenschaftler angehören. Von den Autoren bzw. den Institutionen werden Artikelbearbeitungsgebühren bzw. Autorengebühren erhoben, deren Höhe gegenwärtig zwischen US $\$ 500^{31}$ und US \$ $1500^{32}$ variiert.

\section{BioMed Central ${ }^{33}$}

BioMed Central ist ein Online-Verlag für die Fachgebiete Biologie und Medizin. Der Verlag verfügt derzeit über ein Portfolio von ca. 100 Zeitschriften, die als elektronische Dokumente frei zugänglich sind. Hiermit kommt BioMed Central den Forderungen der Budapest Open Access Initiative nach einem uneingeschränkten Zugang zur wissenschaftlichen Information nach. Ausgewählte Zeitschriften können auf Nachfrage als print-on-demand am Ende des Jahres bestellt werden. Für die Archivierung der digitalen Dokumente kooperiert der Verlag mit PubMed Central, dem nationalen Archiv für elektronische Dokumente aus den Fachgebieten Biologie und Medizin in den USA.

Die Qualitätssicherung erfolgt durch das Peer Review, welches durch die den Gutachtergremien angehörenden Wissenschaftler wahrgenommen wird. BioMed Central stellt den workflow für ein transparentes Peer Review zur Verfügung. Es ist jedoch den Herausgebern und Gutachtern der Zeitschrift überlassen, ob sie das traditionelle doppelt-blinde Verfahren oder transparentere offene Begutachtungsverfahren durchführen.

Für die Finanzierung der Kern- und zusätzlichen Dienstleistungen erhebt BioMedCentral eine Pauschalgebühr von den Autoren in Höhe von US \$ 500 (Stand: 2002). Darüber hinaus bietet der Verlag den Hochschulen und außeruniversitären Forschungseinrichtungen sogenannte institutionelle Mitgliedschaften an. Die Institutionen entrichten eine Gebühr und erwerben auf diese Weise eine Publikationsmöglichkeit für die Wissenschaftler ihrer Einrichtung, ohne dass diese Artikelbearbeitungsgebühren zahlen müssen. Die Höhe der Gebühr für die institutionelle

${ }^{27}$ Vgl. hierzu auch Kuhlen (Anm. 7) S. 34 f.

${ }^{28}$ Kuhlen (Anm. 7) S. 34.

${ }^{29}$ Vgl. hierzu "Werdet Teil der Revolution". Interview mit Harold Varmus vom 18.06.2003. In: Die Zeit 26, 2003. Zugang: <http://zeus.zeit.de/text/2003/26/N-InterviewVarmus> (30.06.03). Vgl. hierzu auch Odlyzko, Andrew: (Anm. 4).

30 Zugang: <http://www.soros.org/openaccess/g/index.shtml>.

${ }^{31}$ BioMedCentral, New Journal of Physics, Molecular Diversity Preservation International Foundation.

32 PLoS Biology, PLoS Medicine.

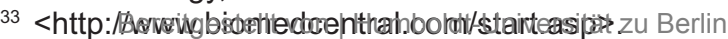


Mitgliedschaft ist abhängig von der Anzahl der Studenten und Postgraduierten der biologischen und medizinischen Fakultät der Hochschule. Sie variiert von US \$1550 pro Jahr für kleine Institutionen mit einer Mitgliedzahl von 20-500 Studenten/Postgraduierten bis zu US \$ 7750 für sehr große Institutionen mit einer Mitgliedzahl von mehr als 5000 Studenten/Postgraduierten. BioMed Central bietet den Wissenschaftlern auch eine Publikation des Artikels auf einer für die Mitgliedsinstitution eingerichteten Webseite an.

Als Kerndienstleistungen bietet der Verlag die Autorenunterstützung bei der Konvertierung der Dokumente in offene Dokumentformate und die Verlinkung der Dokumente in die fachlichen Netzwerke an. Als Mehrwertdienst wird ein Rankingverfahren „Faculty of 1000 “ angeboten. Hierbei handelt es sich um einen post-review Prozess, in dem ein Gremium von Wissenschaftlern in regelmäßigen Abständen die für sie interessantesten Artikel benennen. Diese Mehrwertdienstleistung muss durch Institutionen oder Einzelpersonen subskribiert werden.

Die rechtliche Situation ist zugunsten der Wissenschaftler geregelt. Während die Zeitschrift sich im Besitz des Verlages befindet, verbleiben die Rechte auf die Inhalte und das Copyright beim Autor. Eine Veröffentlichung des Manuskriptes als preprint in einem elektronischen Archiv ist möglich.

\section{New Journal of Physics ${ }^{34}$}

Seit 1997 wird die elektronische Zeitschrift New Journal of Physics ${ }^{35}$ von der Deutschen Gesellschaft für Physik (DGP) und dem Institute of Physics (IOP) herausgegeben. Das IOP verfügt über eine verlegerische Infrastruktur und umfangreiche verlegerische Kompetenzen. In der Kooperation mit einem professionellen Verlagspartner sieht die DGP einen wesentlichen Vorteil, da sie über keine eigene Verlagskompetenz verfügt. Die Konzeption der Zeitschrift sieht vor, dass alle Gebiete der theoretischen und der experimentellen Physik abgedeckt werden. Es findet bewusst keine Fokussierung auf einzelne Schwerpunkte innerhalb der Physik statt. Derzeit sind jedoch noch einige Fachgebiete unterrepräsentiert.

Für die Herausgabe einer Zeitschrift auf der Basis des freien Zugangs zur Information werden vom Vorstand Zeitschriftenbereich der DGP mehrere Gründe genannt ${ }^{36}$ :

Mit Hilfe der neuen Technologien ist eine beschleunigte Publikation der wissenschaftlichen Ergebnisse möglich. Die Bearbeitungszeiten bei der Zeitschrift New Journal of Physics lagen im Jahr 2002 bei 82 Tagen $^{37}$. Das Konzept des freien Zugangs maximiert die Reichweite und Sichtbarkeit der publizierten Artikel. Darüber hinaus bieten die neuen Technologien erweiterte und völlig neue Darstellungsformen. Der Anteil der Nutzung dieser medienspezifischen Vorteile liegt derzeit bei $12 \%$ der publizierten Artikel. Wie bei BioMed Central, wird die Zeitschrift durch Artikelbearbeitungs- bzw. durch Autorengebühren finanziert.

Der ersten Veröffentlichung ging eine längere Phase der Werbung für das neue Konzept der Zeitschrift voraus. Bis zu diesem Zeitpunkt hatte auch das IOP noch keinerlei Erfahrungen mit der Herausgabe einer Zeitschrift auf der Basis dieses neuen Geschäftsmodells. Mittlerweile erhält die Zeitschrift internationale Unterstützung durch 18 weitere Fachgesellschaften, die Autoren auf die neue Publikationsmöglichkeit aufmerksam machen.
Mit dem Wechsel des Chefherausgebers in der Mitte des Jahres 2001 konnte die bis dahin unbefriedigende Publikationssituation stabilisiert werden. Im Jahr 2001 wurden 101 Artikel publiziert. Knapp 50 \% wurden durch Zuschüsse der Gesellschaften finanziert, da nicht alle Autoren die Finanzierung leisten konnten oder wollten.

Die Zeitschrift führt ein Peer Review im doppelt-blinden Verfahren durch. Die Ablehnungsquote liegt derzeit bei $62 \%$. Für die eingereichten Artikel gibt es keine Formatbeschränkungen. Vom Verlag werden die Dokumente in offene XML-Formate konvertiert, die Indizierung und Verlinkung wird vorgenommen.

Als Erfolgsparameter sieht die DGP die Volltextdownloads an, die in den vergangenen Jahren kontinuierlich gestiegen sind. Darüber hinaus soll der Zeitschrift in diesem Jahr erstmals ein Impact Faktor durch das Institute of Scientific Information zugewiesen werden.

\section{Public Library of Science ${ }^{38}$}

Mit finanzieller Unterstützung der Moore-Foundation ${ }^{39}$ hat die Public Library of Science im Herbst des Jahres 2003 eine Fachzeitschrift auf dem Gebiet der Biologie herausgegeben. Die Herausgabe einer weiteren Zeitschrift ist im Frühjahr 2004 vorgesehen. Für die Publikation in diesen Zeitschriften wird von den Autoren bzw. den Institutionen eine Artikelbearbeitungsgebühr erhoben, die eine umgehende Publikation nach dem peer-review (doppeltblindes Verfahren) möglich macht. Die Vorteile des freien Zugangs zur wissenschaftlichen Information formuliert die Public Library of Science in Anlehnung an die Prinzipien der BOAl folgendermaßen:

"Open access publication will:

- Greatly expand access to scientific knowledge by giving any scientist, physician, student - or anyone with access to the Internet, anywhere in the world - unlimited access to the latest scientific research.

- Facilitate research, informed medical practice and education by making it possible to freely search the full text of every published article to locate specific ideas, methods, experimental results and observations.

- Enable scientists, librarians, publishers and entrepreneurs to develop innovative new ways to access and use the information in this immensely rich but highly fragmented resource." 40

Autoren, die zukünftig in den Fachzeitschriften publizieren, werden die Rechte an ihren Arbeiten behalten, so dass Veröffentlichungen in anderen elektronischen Archiven

${ }^{34}<$ http://www.iop.org/EJ/njp>.

${ }^{35}$ Es wird keine Druckversion der Zeitschrift herausgegeben.

36 Telefonat mit Hr.Dr.Georg Botz am 12.05.2003.

37 Hiervon entfallen 37 Tage auf die Entscheidungsphase (Begutachtung), 20 Tage auf die Produktion und 25 Tage auf die Nachbearbeitung durch die Autoren. Im Jahr 1999 betrug die Bearbeitungsdauer pro Artikel noch 130 Tage.

$38<$ http://www.publiclibraryofscience.org/>.

39 Die Moore-Foundation hat eine Summe von US \$ 9 Mio. für die Herausgabe elektronischer Fachzeitschriften gestiftet.

$40<$ http://www.publiclibraryofscience.org/PLoS_Moore PressRedegseteltt7Dec;2002spddf $>$ Universität zu Berlin 
möglich sind. Geplant sind weitere Zeitschriften, die als direkte Konkurrenten zu etablierten Zeitschriften kommerzieller Verlage herausgegeben werden sollen ${ }^{41}$.

\section{Molecular Diversity Preservation International ${ }^{42}$}

Molecular Diversity Preservation International (MDPI) ist ein Projekt in der Schweiz, welches vier Zeitschriften des Fachgebietes Chemie herausgibt ${ }^{43}$. Die seit 1996 erscheinende Zeitschrift Molecules ist bereits in den Zitationsindices des ISI nachgewiesen. Die Finanzierung der Zeitschriften erfolgt über Artikelbearbeitungsgebühren in Höhe von derzeit US \$ 500. Unterstützung erhält das Projekt bzw. die Wissenschaftler durch die Soros-Stiftung in solchen Ländern, in denen die Stiftung aktiv ist ${ }^{44}$. Für diesen Personenkreis übernimmt sie die $100 \%$ ige Finanzierung für zwei Artikel pro Jahr, darüber hinaus erscheinende Publikationen werden zu 50 \% durch die Stiftung finanziert.

Das Gutachtergremium ist international besetzt und die Begutachtung der Manuskripte erfolgt im doppelt-blinden Verfahren. Neben der elektronischen Version sind die Artikel auf einer CD-ROM und in gedruckter Form erhältlich. Für die Publikationen bestehen Partnerschaften mit der französischen Universität ENSTA und der Ocean University in China.

\section{German Medical Science ${ }^{45}$}

Bei German Medical Science (GMS) handelt es sich um ein deutsches Kooperationsprojekt der 140 in der Arbeitsgemeinschaft der Wissenschaftlichen Medizinischen Fachgesellschaften (AWMF) zusammengeschlossenen Mitgliedsgesellschaften, der Zentralbibliothek für Medizin (ZBMed) und des Deutschen Instituts für Medizinische Dokumentation und Information (DIMDI). German Medical Science ist eine elektronische Zeitschrift und ein Portal für elektronische Dokumente aus allen Fachgebieten der Medizin ${ }^{46}$. Ziel ist die weltweite Distribution qualitativ hochwertiger Forschungsergebnisse in deutscher und englischer Sprache. Die GMS bietet folgende Dienstleistungen $\mathrm{an}^{47}$ :

- GMS als Online-Verlag: Veröffentlichung hochrangiger interdisziplinärer und qualitätsgeprüfter Original- und Übersichtsarbeiten aus dem gesamten Spektrum der Medizin,

- elektronische Aufbereitung und Publikation von Zeitschriften einzelner Fachgesellschaften unter deren Titel mit wissenschaftlichen und qualitätsgeprüften Originalarbeiten,

- GMS als Fachportal: Veröffentlichung von Nachrichten, Mitteilungen etc. an die Mitglieder, Informationen über Kongresse, Tagungen etc.

Die Aufgaben, die sich mit der Herausgabe eines elektronischen Journals verbinden, verteilen sich auf die Kooperationspartner folgendermaßen: die AWMF stellt das Editorial Board, die Beiratsmitglieder und die Gutachter für den Begutachtungsprozess, die ZBMed übernimmt die redaktionelle Arbeit und die technische Realisierung und die langfristige Archivierung der Dokumente wird durch DIMDI sichergestellt.

Mit German Medical Science wird der Wissenschaftlergemeinschaft eine Umgebung angeboten, die die Potenziale des elektronischen Publizierens voll ausschöpft. Der Zu- gang zu den elektronischen Dokumenten ist - in Anlehnung an die BOAI - frei (open access). Der Wissenstransfer wird durch das elektronische Publizieren beschleunigt. Der durchschnittliche Zeitraum zwischen dem Einreichen des Dokuments, der Begutachtung und der Veröffentlichung beträgt bei GMS durchschnittlich 6-10 Wochen.

GMS belässt die Rechte an den Dokumenten bei den Autoren. Das Manuskript kann weiterverbreitet werden, der Autor muss die GMS lediglich als erstes Publikationsorgan angeben. Die bei GMS veröffentlichten Dokumente werden in die medizinischen Referenzdatenbanken (z.B. Medline) verlinkt. Darüber hinaus bietet GMS eine Alternative zum Rankingverfahren des ISI an: von GMS werden die Häufigkeiten des Zugriffs auf das elektronische Dokument als Indikator für eine Bewertung der Relevanz gemessen.

Um German Medical Science als Kommunikations- und Publikationsforum zu etablieren, werden gegenwärtig für die Publikation bei GMS noch keine Gebühren erhoben. Das Forum Qualitative Sozialforschung (FQS) ${ }^{48}$ ist ein interdisziplinäres, internationales und mehrsprachiges Forum. FQS ist Herausgeber einer elektronischen Zeitschrift und bietet den entsprechenden Fachdisziplinen ein umfassendes Informationsportal zu dem Themengebiet an. Es trägt auf diese Weise zur Vernetzung zwischen den Disziplinen bei. Im FQS werden nur begutachtete Artikel (doppelt-blindes Verfahren ${ }^{49}$ ) veröffentlicht. FQS ist Herausgeber von Einzelbeiträgen und Beiträgen in den Rubriken Reviews, Debatten und Tagungen. Es werden Buchrezensionen durchgeführt und Newsletters herausgegeben.

\section{Directory of Open Access Journals (DOAJ) ${ }^{50}$}

Durch eine Kooperation der Lund Universität mit der Scholarly Publishing and Academic Resources Coalition (SPARC) und dem Informationsprogramm des Open Society Institute (OSI) der Soros-Stiftung wurde im Frühjahr 2003 ein elektronisches Verzeichnis für wissenschaftliche Fachzeitschriften eröffnet, welches im Sinne der BOAI einen freien Zugang zur wissenschaftlichen Information gewährleisten soll. Mit dem Aufbau eines solchen Verzeichnisses wird das Ziel verfolgt, „[...] to increase the visibility and ease of use of open access scientific journals, thereby promoting their increased usage and impact. The

41 PLoS Computer Science, PLoS Chemistry.

$42<$ http://www.mdpi.org/>.

${ }^{43}$ Molecules, Entropy, Molecular Science, Sensors.

$44<$ <ttp://www.soros.org/>.

$45<$ http://ger-med-sci.de/>. German Medical Science ist seit dem 1.07.2003 online.

${ }^{46}$ Finanziert wird GMS von der Deutschen Forschungsgemeinschaft.

47 Vgl. Richter, Ludwig: German Medical Science. Projektbericht (ohne Datum). Zugang: <http://www.iwi-iuk.org/iuk2003/ program/richter>

48 <http://www.qualitative-research.net/fqs/fqs.htm>. Ein ähnliches Fachportal ist CogNet.

49 Das doppelt-blinde Verfahren zeichnet sich dadurch aus, dass Gutachter und Verfasser einander nicht bekannt sind. Vgl. ausführlicher Kapitel 3.

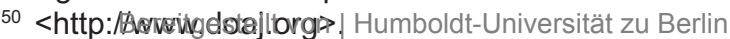


Directory will comprehensively cover all open access scientific journals that use an appropriate quality control system, and it will not be limited to particular languages or subject areas" 51 .

Die Sicherstellung der Begutachtung und eine alternative Finanzierung (durch Autorengebühren, Artikelbearbeitungsgebühren, Spenden etc.) sind die Voraussetzungen für die Aufnahme in das DOAJ-Verzeichnis. Derzeit befinden sich 757 (Stand: 24.02.04) Zeitschriften aus allen Fachgebieten in dem Verzeichnis. Zu den am stärksten vertretenen Fachdisziplinen zählen die Medizin, Biologie, Mathematik, Erziehung und Informatik. Auch die Zeitschriften von BioMed Central sind hier zu finden.

\section{Bewertung}

Das gegenwärtige Geschäftsmodell der Subskription/Lizenzierung/pay-per-view (SLP) hat eine Doppelfinanzierung der wissenschaftlichen Information durch die öffentliche Hand zur Folge. Hochschulen finanzieren die Entwicklung und Produktion wissenschaftlicher Forschungsergebnisse und erwerben die veröffentlichten Ergebnisse in Form der Zeitschriften von den Verlagen zurück. Dies ist die Konsequenz des „Outsourcing" des Publikationsprozesses, wie er sich für die Printumgebung durchgesetzt hat.

In dem neuen Geschäftsmodell verändern sich die Geldströme dahingehend, dass die Hochschule bzw. die der Hochschule angehörenden Wissenschaftler die Kosten für die Produktion und Verbreitung der wissenschaftlichen Information tragen. Dies kann zu einer Steigerung des Kostenbewusstseins für die Publikationsprozesse bei den beteiligten Akteuren beitragen.

Der eigentliche Vorteil des Geschäftsmodells liegt jedoch in der Ausnutzung des Potenzials der neuen Technologien, in der Möglichkeit der globalen Verbreitung und des freien Zugangs zur wissenschaftlichen Information. Die uneingeschränkte Verfügbarkeit der wissenschaftlichen Information ist Voraussetzung für die Produktion neuen Wissens. Hierdurch werden die Partizipationschancen auch für solche Wissenschaftler erhöht, denen bislang nur ein eingeschränkter Zugang zur Information möglich ist.

Die Tragfähigkeit dieses neuen Geschäftsmodells ist noch nicht abschließend zu beurteilen. Eine erfolgreiche Etablierung hängt wesentlich davon ab, ob es gelingt, das elektronische Publizieren bzw. das elektronische Dokument als erste Version jeden Dokumentes zu verbreiten und die Wissenschaftler von den Publikationsalternativen und der Notwendigkeit eines neuen Geschäftsmodells zu überzeugen.

Derzeit finanziert sich keine der genannten Unternehmungen selbst. Neben den bereits genannten Stiftungen, die für die Finanzierung von Zeitschriften aufkommen, wird auch BioMed Central durch einen privaten Geldgeber unterstützt. Auffallend ist, dass die genannten Unternehmungen in dem dynamischen STM-Gebiet ihre Aktivität entfalten. Die Chancen einer Durchsetzung des neuen Geschäftsmodells wird in diesem Gebiet von den Akteuren als am wahrscheinlichsten eingestuft. Einen Einfluss auf das Publikationsverhalten der Wissenschaftler erreichen die Unternehmungen jedoch nur dann, wenn es ihnen gelingt, ihre Zeitschriften als „Markenartikel“ zu etablieren und diese einen hohen Impact Faktor erhalten.

Welche Auswirkungen die erfolgreiche Etablierung der Zeitschriften auf die Preispolitik (insbesondere bei Bio-
Med Central, einem kommerziellen Akteur) hat, lässt sich gegenwärtig noch nicht absehen. Zu Beginn des Jahres 2004 hat BioMed Central die Kosten für die institutionelle Mitgliedschaft erhöht.

\subsection{Verlagsunabhängige Publikationsinfrastruktu- ren - Selbstorganisation der Wissenschaften - Beispiele}

\section{Peer-to-Peer Kommunikation in der Wissenschaft}

Seit Mitte der 90er Jahre wird das Peer-to-Peer (P2P) Netzwerk vor allem im privaten Bereich zum Austausch von Videodokumentationen und MP3-Musikfiles genutzt. Napster gilt heute als der „Urvater" der Nutzer-zu-Nutzer Tauschbörsen. Die öffentliche Aufmerksamkeit konzentrierte sich jedoch in den vergangenen Jahren stärker auf die Fragen des legalen Gebrauchs bzw. des möglichen Missbrauchs dieser Netzwerke und weniger auf die Möglichkeiten, die diese Technologien für andere Felder bieten. Von Steinmetz werden folgende Merkmale der Peer-toPeer Systeme genannt.

- Die relevanten Betriebsmittel befinden sich an den sogenannten „Kanten“ der Rechnernetze, auf den Peers.

- Ein Peer ist in der Regel nicht mit derselben Internetadresse zu identifizieren, sondern die Peers erhalten jeweils eine neue IP-Adresse bei der Zuschaltung auf das Netz (variable Konnektivität).

- Es existiert kein Unterschied zwischen Client-ServerFunktionalitäten, Peers sind beides. Das Potenzial des Peer-to-Peer liegt gerade in der dynamischen Netzstruktur. Die Daten werden direkt, ohne eine zentrale Kontrolle, zwischen den Peers übertragen.

- Zumeist sind die Peers über die ganze Welt verteilt und die vorgehaltenen Daten existieren in Form vielfältiger Replikate.

- Peers bilden eher spontane und sich selbst regulierende Netzwerke. Sie werden nicht zentral koordiniert und verwaltet. $^{52}$

Nach Mauthe und Hutchison kommen die Peer-to-Peer Systeme vor allem auf den Feldern des „[...] file and content sharing; collaboration support, distributed computing, communication and plattforms [...] ${ }^{\text {"53 }}$ zur Anwendung. Die Autoren ordnen den Peer-to-Peer Systemen folgende Strukturmerkmale zu:

- Dezentralität

Speicherung, Datenverarbeitung und Informationsaustausch erfolgt dezentral ohne einen zentralen Einstiegspunkt.

- Selbstorganisation

Die verschiedenen beteiligten Systeme arbeiten selbstorganisierend in dem Sinne, dass für die erforderlichen Abläufe kein zentrales Management mit festgelegten Rollen und Anforderungen notwendig ist.

$51<$ <ttp://www.doaj.org/articles/news/030214>.

52 Vgl. Steinmetz, Ralf: Peer-to-Peer: Maßlos verkannt! (2003). Zugang: <http://www.multimedia-communications.net/ meinung.php?s=\&meinungs id=61> (21.07.03).

53 Mauthe, Andreas; Hutchison, David: Peer-to-Peer Computing: Systems, Concepts and Characteristics. In: Praxis der Informationsverarbeitung und Kommunikation (PIK) 26,

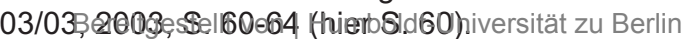


- Fehlertoleranz

Bedingt durch ihre Struktur und ihre Organisation verfügen die Peer-to-Peer Systeme über eine gewisse Fehlertoleranz. Die Funktionen werden auch bei dem Ausfall einiger Peers weiterhin aufrechterhalten.

- Skalabilität

Die Systemskalabilität wird durch das Fehlen zentraler Server nicht maßgeblich beeinträchtigt ${ }^{54}$.

In den Peer-to-Peer Systemen ist jeder Teilnehmer gleichberechtigt, von jedem Teilnehmer werden Dienste zur Verfügung gestellt und Dienste von anderen in Anspruch genommen. Jeder Nutzer stellt nach dem Einloggen Files auf seiner eigenen Festplatte anderen Nutzern zur Verfügung. Im Gegenzug gewinnt er Zugriff auf die Dateien der anderen momentan eingeloggten Teilnehmer. Durch die globale Verteilung der Peers ist die Entwicklung geeigneter Sicherungskonzepte eine wichtige Aufgabe.

Um diese Technologie für die Wissenschaft nutzbar zu machen, wird die Entwicklung einer prototypischen Peer-toPeer Volltext-Suchmaschine vom Deutschen Forschungsnetz (DFN) für einen Zeitraum von zwei Jahren mit 450000 Euro finanziell unterstützt ${ }^{55}$. Das DFN-Science-to-Science wird auf einem Peer-to-Peer Netzwerk basieren, das von Wissenschaftlergemeinschaften betrieben wird. Das Gigabit-Wissenschaftsnetz (G-WIN) bildet die Grundlage zum effizienten Austausch wissenschaftlicher Informationen ${ }^{56}$. Science-to-Science unterscheidet sich von anderen Peerto-Peer-Netzwerken, wie z.B. Gnutella, Kazaa (die in der Nachfolge von Napster entstanden sind) dadurch, dass nicht jeder Nutzer selbst Inhalte im Netzwerk anbieten muss.

Die Suche erfolgt bei Science-to-Science im Volltext und mit Hilfe der Metadaten der erfassten wissenschaftlichen Dokumente ${ }^{57}$. Die hierfür entwickelte Suchmaschine JXTA referenziert auch Dokumente in Literaturlisten, Datenbankeinträge etc., also Informationen im sogenannten Deep Web. Neofonie, der Projektbeauftragte des DFN sieht die Vorteile dieser Netzwerke in der höheren Aktualität (kein zentraler Index), dem schnelleren Austausch wissenschaftlicher Information, den uneingeschränkten Publikationsmöglichkeiten und dem gleichberechtigten Zugang zur Information. Als nachteilig wird die fehlende Wiederauffindbarkeit der Dokumente in den Netzwerken kritisiert. Darüber hinaus sind umfangreiche Filtermaßnahmen zur Abwehr von SPAM bzw. illegalem Inhalt erforderlich.

Derzeit ist Peer-to-Peer noch eine sich entwickelnde Technologie. Ihr Potenzial für den wissenschaftlichen Kommunikationsprozess liegt insbesondere in der Beschleunigung der Kommunikation wissenschaftlicher Gemeinschaften bzw. Arbeitsgruppen.

\section{Elektronische Archive - Repositorien}

Bereits seit einigen Jahren lässt sich international der Aufbau verlagsunabhängiger Kommunikations- und Publikationsinfrastrukturen beobachten. Die Entwicklung dieser institutionellen Repositorien erfolgt auf der Ebene einzelner Fachgebiete und auf der Ebene einer bzw. mehrerer Hochschulen. Diese elektronischen Archive basieren auf dem Ansatz der Selbstarchivierung und sind durch den Einsatz des OAI-Protokolls über Suchmaschinen recherchierbar ${ }^{58}$. Einen Überblick über die Entwicklungen der elektronischen Archive in den USA bietet SPARC. Auf europäischer Ebene bietet das Open Archives Forum einen Überblick ${ }^{59}$, ein Projekt, das die existierenden Initiativen dokumentiert und sich als Forum für den Austausch zwischen den beteiligten Akteuren versteht. Derzeit ist die Anzahl der Institutionen, die den Aufbau dieser Publikationsinfrastrukturen vorantreiben, in England und Deutschland am größten ${ }^{60}$.

\section{Repositorien auf fachlicher Ebene ${ }^{61}$}

Die Bedeutung der fachlichen Repositorien liegt vor allem in der Beschleunigung der Kommunikationsprozesse innerhalb einer Fachdisziplin. Die Manuskripte werden vor der Veröffentlichung in einer Fachzeitschrift auf einem Server öffentlich zugänglich gemacht. Da diese (noch) nicht in einer Zeitschrift publiziert werden, sind sie frei von rechtlichen Restriktionen durch die Verlage. Die preprints sind jedoch nicht durch ein Begutachtungsverfahren in ihrer Qualität bewertet worden.

Das bekannteste Beispiel für ein fachliches Repositorium ist der 1991 entwickelte Dokumentenserver ArXiv62, ein Server für preprints aus dem Fachgebiet der Physik, der durch die Privatinitiative von Paul Ginsparg entstanden ist. In einem automatisierten Einreichungsprozess werden die Forschungsergebnisse vor der eigentlichen Veröffentlichung allgemein zugänglich gemacht. Meier weist darauf hin, dass es in der Physik allgemein übliche Praxis ist, eine mit der Veröffentlichung identische Version des Artikels auf dem ArXiv-Server zu stellen. Dies kann für andere Wissenschaftsbereiche von Interesse sein, in denen Verlage nach wie vor rigide Verwertungsrechte an dem Artikel geltend machen ${ }^{63}$.

CogPrints ${ }^{64}$ wurde nach dem Vorbild des ArXiv-Servers für die verschiedenen Fachdisziplinen der Kognitionswissenschaften entwickelt. Hierzu zählen: Psychologie, Neurowissenschaften, Biologie, Informatik, Linguistik und Philosophie. Der Preprint-Server RePEC ${ }^{65}$ enthält

${ }^{54}$ Mauthe; Hutchison (Anm. 53) S. 61.

${ }^{55} \mathrm{Vgl}$. <http://www.neofonie.de/profil/forschung_und_entwicklung/ s2s.csp> (21.06.03).

${ }^{56} \mathrm{Vgl}$. <http://www.innovationsreport.de/html/berichte/ kommunikation_medien/bericht-10224.html> (21.06.03).

${ }^{57}$ Ebd.

${ }^{58}$ Elektronische Archive werden auch als Data Provider bezeichnet. Neben den institutionellen Repositorien fallen "Subject Gateways", Online Public Access Catalogues und auch die Datenbanken der kommerziellen Verlage etc.in diese Kategorie. Sie werden in diesem Zusammenhang jedoch nicht weiter berücksichtigt.

59 Vgl. hierzu auch: <http://www.oaforum.org/>.

${ }^{60}$ Vgl. ausführlicher Dobratz, Susanne; Matthaei, Birgit: Open Archives Activities and Experiences in Europe. An overview by the Open Archives Forum. In: D-Lib Magazine, Vol. 9, 2003, Nr. 1. Zugang: <http://www.dlib.org/dlib/january03/ dobratz/01dobratz.html> (14.06.03).

${ }^{61}$ Exemplarische Darstellung einiger fachlicher Repositorien.

$62<$ http://arxiv.org/>.

${ }^{63}$ Vgl. Meier (Anm. 8) S. 157

$64<$ http://cogprints.ecs.soton.ac.uk/>

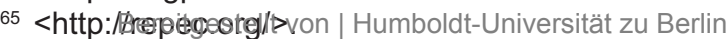

Angemeldet 
Manuskripte aus dem Fachgebiet der Wirtschaftswissenschaften.

Während es sich bei den genannten Beispielen um einen an einer Universität angesiedelten Server handelt, basiert die Konzeption von MPRESS ${ }^{66}$ auf einem dezentralen Ansatz. Die preprints des Fachgebietes Mathematik verbleiben auf den Servern, die vom Autor gewünscht werden. MPRESS sucht die Server auf der Basis von Metadatensätzen ab und erstellt einen automatischen Index.

Nicht nur die Beschleunigung und Vereinfachung des wissenschaftlichen Austauschs sind Vorteile, die mit den fachlichen Repositorien realisiert werden. Sie erleichtern den beteiligten Wissenschaftlern des Fachgebietes die Identifikation mit dem Archiv und ermöglichen eine eindeutigere Vorstellung vom Inhalt des elektronischen Archivs. Eine Erweiterung der fachlichen Respositorien stellen die institutionellen Repositorien dar, die als elektronische Archive der Hochschulen entwickelt werden.

\section{Repositorien auf institutioneller Ebene}

Das Konzept der institutionellen Repositorien bezieht die Fachgebiete der gesamten Institution ein. Für die beteiligten Abteilungen, Institute, Forschungsprojekte etc. wird eine elektronische Publikationsumgebung aufgebaut, die einen Zugang unter einer einheitlichen Oberfläche gewährleistet.

Von mehreren Initiativen bzw. Gremien ist auf die strategische Bedeutung der institutionellen Repositorien für die Reformierung des Systems wissenschaftlicher Kommunikation hingewiesen worden. Die Scholarly Publishing \& Academic Resources Coalition (SPARC) hat im vergangenen Jahr ein Positionspapier herausgegeben, welches den Aufbau wissenschaftseigener Publikationsinfrastrukturen empfiehlt ${ }^{67}$. Institutionelle Repositorien werden definiert als ein ,[...] digital archive of the intellectual product created by the faculty, research staff, and students of an institution and accessible to end users both within and outside of the institution, with few if any barriers to access" ${ }^{\prime 6}$. Sie kennzeichnen sich durch:

- ihre institutionelle Ausrichtung:

Im Gegensatz zu den disziplinspezifischen Archiven umfasst das institutionelle Repositorium sämtliche an der Hochschule vertretenen Disziplinen. Mit innen wird der intellektuelle „Output" der jeweiligen Institution öffentlich zugänglich gemacht. Da die Qualitätssicherung durch die Institution bzw. die akademischen Strukturen sichergestellt werden, sind die Archive zugleich ein Indikator für die akademische Qualität der Hochschule.

- ihren wissenschaftlichen Inhalt:

preprints, working papers, Forschungsergebnisse, publizierte Artikel, Lern- und Lehrmaterialien und Qualifikationsarbeiten.

- Wachstum und Dauerhaftigkeit:

Die Entwicklung von Kriterien, Grundsätzen und Zugriffsrechten für die Archivierung und den Zugriff sind grundlegend für den erfolgreichen Aufbau und die dauerhafte Bereitstellung der Inhalte.

- Interoperabilität und freier Zugriff:

Das Ziel des Aufbaus eines institutionellen Repositoriums liegt in der Sichtbarkeit und Verbreitung der von der Hochschule produzierten Materialien. Dafür bildet der freie Zugriff auf die Inhalte des elektronischen Archivs die Voraussetzung. ${ }^{69}$
In den Repositorien befinden sich Ergebnisse wissenschaftlicher Lehre und Forschung, deren Qualität durch die Prüfungsordnungen der Hochschulen bzw. durch die Wissenschaftler selbst abgesichert sind sowie Manuskripte, die noch nicht begutachtet wurden. Die Weiterentwicklung der Begutachtungsverfahren für diese elektronischen Archive stellt eine wichtige Zukunftsaufgabe dar.

In Deutschland wird der Aufbau neuer Publikationsinfrastrukturen von der Hochschulrektorenkonferenz (HRK) ausdrücklich empfohlen. Den Repositorien wird eine zentrale Rolle für die strukturelle Reform der traditionellen Wertschöpfungskette wissenschaftlicher Information zugewiesen. Nach Auffassung der HRK lässt sich das bestehende Ungleichgewicht zwischen Verlagen und Hochschulen in der Wertschöpfungskette wissenschaftlicher Information mit dem Aufbau von elektronischen Archiven zugunsten der Hochschulen abschwächen. Diese geben die mit dem Publikationsprozess verbundenen Aufgaben nicht länger an die Verlage ab, sondern nehmen die Produktion, Distribution und Archivierung der Ergebnisse aus Lehre und Forschung selbst in die Hand ${ }^{70}$. Im folgenden Kapitel werden die Konzeptionen verschiedener institutioneller Repositorien beschrieben.

\section{Max-Planck-Gesellschaft/e-Doc-Server}

Das Zentrum für Informationsmanagement der Heinz Nixdorf Stiftung (ZIM) unterstützt die der Max-PlanckGesellschaft angehörenden Institute in dem Prozess elektronischer Kommunikation und Publikation. Zu diesem Zweck hat das ZIM ein institutionelles Repositorium für die Aufbewahrung, das Management, die Verbreitung, die Publikation und Langzeitarchivierung der digitalen Dokumente aufgebaut. Der e-Doc-Server ist in enger Kooperation mit dem Fritz Haber Institut und einer Reihe von Piloteinrichtungen der Max-Planck-Gesellschaft entwickelt worden. Die Institute können den Server zur Unterstützung gemeinschaftlicher Arbeiten nutzen, zur Herausgabe digitaler Publikationen, für die Bereitstellung wissenschaftlicher Materialien, zur Beschleunigung der Kommunikation der Forschungsergebnisse und zur Archivierung der Ergebnisse. Aus institutioneller Sicht verbinden sich mit dem Aufbau des elektronischen Archivs folgende strategische Zielsetzungen:

- „build a comprehensive resource for scientific information produced by the Max Planck Institutes, providing a stable location for its preservation and dissemination,

${ }^{66}<$ http://mathnet.preprints.org/>

${ }_{67} \mathrm{Vgl}$. Crow (Anm. 6).

${ }^{68}$ Vgl. Crow (Anm. 6) S. 16.

${ }^{69}$ Crow (Anm. 6).

${ }^{70}$ Für die Hochschulrektorenkonferenz stellt sich die Struktur des wissenschaftlichen Publikationswesens in Zukunft als ein verteiltes System dar, in dem die Hochschulen ihre wissenschaftlichen Publikationen auf den eigenen Servern halten und die Verlage vorrangig die Aufgabe haben, komfortable Zugangswege und hochwertige Verknüpfungen mit anderen Quellen herzustellen. Zugang: <http://www.hrk.de/

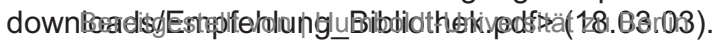


- increase the visibility of the intellectual output of the Max Planck Institutes in all the forms it takes in the era of the internet,

- strengthen the Society and the scientific 'community' in negotiations with publishers about the ownership of scientific research documents at a time where sky-rocketing journal prices and restrictive wide dissemination and persistent accessibility,

- contribute to a worldwide, emerging scholarly communication system, which exploits the full potential of the internet and the digital representation and processing of scientific information."71

\section{Eidgenössische Technische Hochschule Zürich - ETH-E-Collection ${ }^{72}$}

Die Bibliothek der Eidgenössischen Technischen Hochschule in der Schweiz hat im Rahmen eines Großprojekts einen hochschuleigenen Dokumentenserver aufgebaut, der im Jahr 2003 in den Routinebetrieb überführt wurde. Mit dem Aufbau des elektronischen Archivs sollen die bislang in Abteilungen, Instituten und von Einzelpersonen verstreut vorgehaltenen Dokumente unter einer einheitlichen Oberfläche zentral verwaltet und zugänglich gemacht werden. Wissenschaftler erhalten die Möglichkeit, ihre Publikationen umgehend einer breiten Öffentlichkeit zur Verfügung zu stellen ${ }^{73}$. Das elektronische Archiv enthält Dissertationen, Lehrmaterialien, Reports, Tagungsberichte, Zeitschriften, Jahresberichte und Qualifikationsarbeiten. Eine Suche nach Sachgruppen, nach Abteilungen und Autoren ist möglich.

\section{eScholarship}

eScholarship ist ein Kooperationsprojekt der California Digital Library (CDL), der University of California und der Initiative SPARC. Es bietet eine strukturierte Publikations- und Archivierungsumgebung für wissenschaftliche Produktionen an, die größtenteils frei zugänglich sind ${ }^{74}$. eScholarship strukturiert sich in vier Bereiche:

Die "digital repositories" bieten derzeit 50 Gruppen von Wissenschaftlern eines Instituts, einer Abteilung, eines Zentrums oder einer Forschungsgruppe eine Publikationsplattform für die Veröffentlichung der wissenschaftlichen Ergebnisse. Es bestehen Suchmöglichkeiten nach dem Jahr der Veröffentlichung und dem Autorennamen. Die Papiere sind im Volltext zugänglich und können als Empfehlung per e-Mail direkt an Kollegen weitergeschickt werden.

In Kooperation mit Berkeley Electronic Press, einem Online-Verlag, tritt eScholarship auch als Herausgeber elektronischer und frei zugänglicher Zeitschriften auf und bietet interessierten Wissenschaftlern eine Publikationsumgebung mit einem doppelt-blinden Begutachtungsprozess an. Der Universitätsverlag bietet derzeit etwa 500 Monographien in digitaler Form an. Weiterhin bietet eScholarship für die Fachgebiete Geschichte und Humanwissenschaften multimedial aufbereitete Publikationen an.

Nach Erfahrung der California Digital Library spielen die Einfachheit der Bedienung und die Gestaltung der Seiten eine zentrale Rolle für die erfolgreiche Etablierung der elektronischen Archive an der Hochschule. Aus der Sicht der CDL sind die Wissenschaftler die Träger der
Entwicklung alternativer Modelle der wissenschaftlichen Kommunikation. Deren Bedürfnisse sind grundlegend für die konzeptionelle Gestaltung der elektronischen Archive. Von der CDL wurde die Entwicklung der elektronischen Publikationsumgebungen mit solchen Fakultäten begonnen, die ein besonderes Interesse an der Entwicklung neuer Kommunikationsformen zeigten. Die Zurückhaltung einiger Wissenschaftler wurde mit dem fehlenden technischen Verständnis und der Befürchtung eines zu hohen zeitlichen Aufwands begründet.

\section{DSpace}

DSpace ist eine open source Software, die in Kooperation zwischen dem Massachusetts Institute of Technology (MIT) und Hewlett Packard (HP) entwickelt wurde ${ }^{75}$. Für den laufenden Betrieb sind die Bibliotheken des MIT verantwortlich. Zielsetzung von DSpace ,[...] is to encourage widespread adoption of the system among faculty and researchers who submit their digital objects" ${ }^{\text {"6. DSpace }}$ ermöglicht die Bearbeitung und Archivierung verschiedenster Dokumenttypen, z.B. Artikel (Preprints, working papers, technische Berichte, Konferenzpapiere), Bücher, Qualifikationsarbeiten, Computerprogramme, Visualisierungen, Simulationen und andere Modelle, Multimediaprodukte und Objekte für die Lehre.

Um die Akzeptanz des elektronischen Archivs bei den Wissenschaftlern zu erhöhen, bietet DSpace kostenlose Kerndienstleistungen für alle registrierten Mitglieder an. Hierbei handelt es sich um „Interactive Services“ und „Operations Services“. Die „Interactive Services" umfassen die Funktionalitäten, die für den Zugang und das Einreichen von Dokumenten erforderlich sind. Mit den „Operations Services" sind Dienstleistungen gemeint, die im Zusammenhang mit der Archivierung und der langfristigen Verfügbarkeit der Daten stehen, die die Beratung über und die Anwendung des institutionellen Archivs umfassen sowie Informationen, die die Verwaltung der digitalen Sammlungen durch die Wissenschaftlergemeinschaft erleichtern. Hierzu zählt auch ein webbasierter oder telefonischer Auskunftsdienst für die registrierten Mitglieder der „DSpace-Gemeinschaft“.

Darüber hinaus plant DSpace das Angebot von Premiumdienstleistungen, die jedoch kostenpflichtig sind. „The primary aim of Premium Services is therefore not to generate funds but rather to allow MIT Libraries to respond to user needs "77. Als Premiumdienstleistungen werden genannt: - E-Conversion-Services (Digitalisierung, Konvertierung von Dokumentformaten),

\footnotetext{
$71<$ http://edoc.mpg.de/doc/help/edoc_info.epl>.

$72<$ http://e-collection.ethbib.ethz.ch/>.

$73<$ http://www.ethbib.ethz.ch/projekt/ecollection.html>.
}

${ }^{74}$ Zum Teil sind sie auch nur für die Angehörigen der Universität zugänglich.

75 <http://www.hpl.hp.com/mit/>. Weitere Informationen über das Konzept und die Kosten: <http://www.arl.org/lR/barton/ sld008.htm>.

${ }^{76}$ Barton, Mary R.; Harford-Walker, Julie: MIT Libraries `Dspace Business Plan Project. Final Report to the Andrew W. MelIon Foundation, 2002, S. 18.Zugang: <http://www.dspace. $\mathrm{org} / \mathrm{mit} / \mathrm{mellon}$.pdf> (18.02.03).

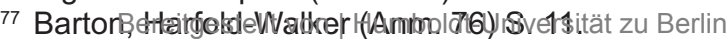


- Metadata Services (administrative, strukturelle, technische, rechtliche Aspekte),

- Costum Repository Services: Überwachung der Veröffentlichungspraktiken innerhalb der Wissenschaftlergemeinschaft durch die Festlegung von Normen zur Größe und Anzahl der Items pro Jahr und Mitglied,

- User Reporting Services: Informationsdienstleistungen für Mitglieder, durch Profildienste, Zitationsauswertungen, Informationen über die Größe, Nutzung, Inhalt der Sammlungen, Anzahl der Artikel und Downloads/Wissenschaftlergemeinschaft oder Sammlung, Anzahl der Besichtigungen der Seiten ${ }^{78}$

Mit der Einführung der neuen Software am MIT geht eine umfassende Informationskampagne einher, die Informationsveranstaltungen und Demonstrationen der elektronischen Publikationsumgebung und Publikationen einschließt. Die schnelle und umfassende Aufbereitung und Bereitstellung der digitalisierten Informationen durch das Bibliothekspersonal wird als kritischer Erfolgsfaktor für die weitere Akzeptanz dieses neuen Mediums angesehen. Zur Unterstützung der Nutzer und zur Weiterbildung des bibliothekarischen Personals sowie zur Weiterentwicklung des Dienstleistungsspektrums wurden deshalb zwei zusätzliche Stellen geschaffen.

\section{Securing a Hybrid Environment for Research Preser- vation and Access - SHERPA ${ }^{79}$}

SHERPA ist ein dreijähriges Projekt, das von dem Konsortium der englischen Universitätsbibliotheken (CURL) finanziert wird und unter Leitung der Universität von Nottingham durchgeführt wird. Es handelt sich um eine Kooperation zwischen sieben Universitäten ${ }^{80}$, der British Library und dem Arts und Humanities Data Service mit dem Ziel, zahlreiche institutionelle e-print-Archive auf der Basis der eprints.org.-Software ${ }^{81}$ aufzubauen. Auslöser für die Entwicklung der elektronischen Archive ist eine zunehmend kritische Haltung gegenüber der wissenschaftsfeindlichen Preispolitik der Verlage. Von Stephen Pinfield, dem Direktor von SHERPA wird dies folgendermassen formuliert: „The current system run by publishers is not working and has not adapted to electronic publishing. Access and impact barriers were accepted in the print-only era as not only were distribution and printing costs high but research could not be distributed to wide audiences due to obvious dissemination restrictions. Nevertheless, in a new electronic environment information can be made available to all at very little cost and with great ease. Publishers need to accept this and change their current system. SHERPA is aimed at investigating ways around these discrepancies." 82

Pinfield sieht das Innovationspotenzial der neuen Technologien insbesondere in der schnellen Verbreitung und der Reduzierung der Kosten für die Produktion und Distribution der wissenschaftlichen Information. Die Initiatoren von SHERPA streben zukünftig Verhandlungen mit Verlagen an, die es Wissenschaftler ermöglichen sollen, ihre Arbeiten in einem nicht-kommerziellen Archiv zu veröffentlichen.

\section{Digital Academic Repositories - DARE ${ }^{83}$}

Digital Academic Repositories (DARE) ist ein vierjähriges nationales Projekt (2003-2006) der niederländischen Universitäten unter Mitarbeit der Koninklijken Bibliotheek (Königliche Bibliothek), der Koninklijken Nederlandse Aca- demie van Wetenschappen (Königliche Niederländische Akademie der Wissenschaften) und der Nederlandse Organisatie voor Wetenschappelijk Onderzoek (Niederländische Organisation für wissenschaftliche Forschung).

Ziel von DARE ist die Modernisierung der niederländischen Informationsinfrastruktur durch den Aufbau digitaler Repositorien, mit denen der wissenschaftliche "Output" der Hochschulen elektronisch zugänglich und langfristig verfügbar gemacht werden soll. Unter Anwendung der internationalen Standards bilden die institutionellen Repositorien eine digitale Plattform für die akademischen Institutionen und sollen dazu beitragen, das Ansehen der niederländischen Hochschulen im internationalen Wettbewerb zu erhöhen. Das verteilte Netzwerk der Repositorien wird working papers, preprints, Forschungsergebnisse, Konferenzveröffentlichungen, Multimediapräsentationen, Dissertationen und andere Qualifikationsarbeiten enthalten. Die elektronischen Archive bilden die Grundlage für die Schaffung von Mehrwertdiensten, die durch die beteiligten Institutionen selbst bzw. in Kooperation mit kommerziellen und nicht-kommerziellen Partnern entwickelt werden sollen.

\section{Bewertung}

Der Aufbau fachlicher und institutioneller Repositorien bietet mittelfristig das größte Potenzial zur Reformierung des wissenschaftlichen Publikationswesens. Hiermit wird der Grundstein für die Entwicklung eines neuen Publikationsmodells gelegt und eine Veränderung in der Wertschöpfungskette wissenschaftlicher Information eingeleitet. Hochschulen übernehmen im elektronischen Umfeld die Funktionen der Produktion und Verbreitung wissenschaftlicher Information.

Die Qualitätssicherung erfolgt derzeit durch

- die existierenden Prüfungsordnungen der jeweiligen Hochschule,

- durch automatisierte Selektionsverfahren nach definierten Minimalkriterien (Preprint-Server).

Eine langfristige Finanzierung der elektronischen Publikationsumgebungen durch die Hochschule ist wünschenswert. $\mathrm{Ob}$ die Hochschulen ihre elektronische Infrastruktur in Zukunft als Bestandteil der Basisinfrastruktur finanzieren werden oder die Bibliotheken (als Träger der Repositorien) den beteiligten Fakultäten die von innen erbrachten Dienstleistungen in Rechnung stellen werden, wie dies bei DSpace der Fall ist, ist derzeit noch offen ${ }^{84}$.

${ }^{78}$ Barton, Harfold-Walker (Anm. 76) S. 13.

$79<$ http://www.sherpa.ac.uk/documents/proposal.pdf>

${ }^{80}$ University of Nottingham (Projektleitung), University of Edinburgh, University of Glasgow, Universities of Leeds, Sheffield and York, University of Oxford.

${ }^{81}$ Eprints-org wurde an der Universität von Southampton für die Selbstarchivierung von wissenschaftlichen Dokumenten entwickelt.

82 Pinfield, Stephen: Open Archives Initiative. Market revolution or hot air? An Electronic Publishing Services (eps) Briefing report. $2003<$ http://www.epsitd.com/>.

83 <http://www.surf.nl/download/DARE-summary.pdf>.

${ }^{84} \mathrm{Vgl}$. hierzu auch Tennant, Roy: Institutional Repositories. Library Journal. (2002). Zugang: <http://www.libraryjournal. reviewsnews.com/index.asp?layout=articlePrint\&articleID= CA242897эe(30).08.02)-Jumboldt-Universität zu Berlin 
Roosendaal, Geurts und van de Vet betonen die Bedeutung der institutionellen Repositorien für die Entwicklung eines Marktes wissenschaftlicher Information zwischen Hochschulen, Verlagen und anderen Abnehmern wissenschaftlicher Information ${ }^{85}$.

In einer strategischen Perspektive nehmen die Hochschulen nicht länger nur die Rolle als Abnehmer, sondern auch als Produzent und Distributor wissenschaftlicher Information ein. Mit dem Aufbau vollständig ausgebauter Publikations- und Archivierungsumgebungen für die Ergebnisse aus Lehre und Forschung übernehmen die Hochschulen Funktionen, die bislang von den Verlagen wahrgenommen werden. Die Position der Hochschule wird vor allem dadurch gestärkt, dass sie die Erstfassung der wissenschaftlichen Ergebnisse aus Lehre und Forschung auf ihrem Server hält. Für die Entwicklung eines Marktes wissenschaftlicher Information sind jedoch entsprechende rechtliche Rahmenbedingungen zwischen Autoren und Hochschulen und die Entwicklung geeigneter Geschäftsmodelle für den Austausch der "Wissensprodukte" von grundlegender Bedeutung. In einer mittelfristigen Perspektive sehen die Autoren die Rolle der Verlage in der Erbringung und Weiterentwicklung von Mehrwertdiensten für die in den Repositorien archivierte wissenschaftliche Information.

\subsection{Neue Kooperationsformen zwischen den Akteuren in der Wertschöpfungskette - Beispiele}

Fachgesellschaften, als Herausgeber von Fachzeitschriften, verfügen häufig nicht über eine geeignete technische Infrastruktur bzw. über das technische Know-how, um ihre Produkte über den Distributionskanal Internet zu vertreiben. Auf dem Gebiet des elektronischen Publizierens lassen sich zahlreiche Kooperationen zwischen Fachgesellschaften, Bibliotheken, Verlagen etc. beobachten.

\section{HighWire Press}

HighWirePress ist ein Online-Verlag, der 1995 von der Stanford University Library gegründet wurde. Er verfügt über ein Zeitschriftenportfolio von derzeit etwa 300 Zeitschriften der Fachgebiete Biologie, Medizin und Lebenswissenschaften. Einige der von HighWirePress herausgegebenen Zeitschriften verfügen über hohe Zitationsindices in den Rankinglisten des ISI. Bei dem Verlag handelt es sich um einen Service Provider, der es zahlreichen Fachgesellschaften ermöglicht, ihre Fachzeitschriften online bereitzustellen. HighWirePress unterstützt die verlegerischen Tätigkeiten dieser Fachgesellschaften durch diverse Dienstleistungen. Einige davon sind:

- die technische Aufbereitung und Konvertierung in offene Dokumentformate,

- die Präsentation und Aufbereitung der Artikel und der Zeitschriften,

- die Beratung bei Geschäftsmodellen,

- das Hyperlinking und Crossreferencing der Artikel in die fachlichen Netzwerke.

Die in HighWire zusammengeschlossenen Fachgesellschaften wenden derzeit überwiegend das traditionelle Subskriptionsmodell in gekoppelter Form an. Darüber hinaus wird ein Pay-per-view und ein Pay-per-site angeboten. Der kostenlose Zugriff auf die zurückliegenden Jahrgänge ist eingeschränkt möglich. HighWire Press bietet ein großes Spektrum an archivierten Artikeln im Volltext an. Die Retrodigitalisierung vergangener Zeitschriftenexemplare soll in Zukunft mit Hilfe einer Stiftung finanziert werden.

Ziel von HighWirePress ist es, als Non-Profit-Organisation auf dem Markt wissenschaftlicher Information wettbewerbsfähig und unabhängig zu bleiben. Von Michael A. Keller, dem Geschäftsführer des Verlages, wird die enge Kooperation mit den Fachgesellschaften als der richtige Weg angesehen, um wissenschaftliche Information bedarfsgerecht für die Wissenschaftlergemeinschaft zu produzieren und zu distribuieren.

\section{ProjectMUSE}

Mit ProjectMUSE bietet der Universitätsverlag der Johns Hopkins University in Kooperation mit der Milton S. Eisenhower Bibliothek elektronische Ausgaben der in dem Verlag erscheinenden und von Fachgesellschaften herausgegebenen Fachzeitschriften aus den Fachgebieten der Sozial- und Geisteswissenschaften und der Mathematik an. Das Portfolio an Zeitschriften umfasst derzeit etwa 200 Zeitschriften. Der Verlag erhält keine finanzielle Unterstützung von der Universität, er arbeitet auf der Basis der Kostendeckung. Als Publikationspartner der Fachgesellschaften übernimmt der Verlag das Marketing, die Websitegestaltung und den Vertrieb der angebotenen Zeitschriften. Das Geschäftsmodell des Johns Hopkins Universitätsverlags beruht auf dem traditionellen Subskriptionsmodell, jedoch werden die Zeitschriften zu wissenschaftsfreundlichen Preisen angeboten. Der Verlag operiert mit verschiedenen Varianten des Subskriptionsmodells und bietet unterschiedliche Pakete an.

\section{BioOne}

Das im Jahr 2000 initiierte Projekt BioOne ist eine Kooperation zwischen einem Online-Verlag, einer Marketing-Agentur, einem Bibliothekskonsortium mit 23 angeschlossenen Bibliotheken und Fachgesellschaften ${ }^{86}$, die die Zeitschriften des American Institute of Biological Science online herausgeben. BioOne verfügt derzeit über ein Portfolio von 55 Zeitschriften, die überwiegend auf Subskriptionsbasis erhältlich sind. Der Online-Verlag bietet den Fachgesellschaften eine elektronische Publikationsumgebung für die Herausgabe und den Vertrieb ihrer Zeitschriften an. Der Internet-Auftritt und die Vermarktung der Zeitschriften wird unterstützt von der Agentur. An der Finanzierung des Projektes BioOne hat sich ein Bibliothekskonsortium beteiligt, das nach Abschluss der Entwicklungsphase die Produkte von BioOne zu günstigen Konditionen bezieht. Für die Verlage und Fachgesellschaften ist dadurch eine

85 Vgl. Roosendaal, Hans; Geurts, Peter A. T. M. und Paul E. van der Vet: Eine neue Wertschöpfungskette für den Markt wissenschaftlicher Information? In: Bibliothek. Forschung und Praxis. 26 (2002) 2, S. 149-154.

86 Vgl. hierzu Johnson, Richard K.: A Question of Access. SPARC, BioOne, and Society-Driven Electronic Publishing. In: D-Lib Magazine 6 (2000) 5. Zugang: <http://www.dlib.org/

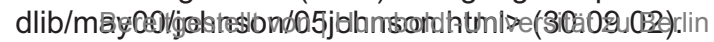


verlässliche Abonnentenbasis vorhanden. Weitere Ansätze zur Finanzierung der Online-Publikationen sind Werbung auf den Seiten von BioOne, Sponsoring etc. BioOne bietet die Verlinkung der publizierten Artikel in die fachlichen Netzwerke an (z.B. BIOSIS; NBII). Der Ausbau dieses Angebotes ist geplant ${ }^{87}$. Für die Archivierung kooperiert BioOne mit PubMedCentral. Darüber hinaus ist die Digitalisierung älterer Zeitschriftenexemplare geplant.

Um die Idee des „open access“ zu realisieren, werden neben dem traditionellen Subskriptionsmodell derzeit drei Zeitschriften frei zugänglich angeboten. Die Herausgabe weiterer frei zugänglicher Zeitschriften auf der Basis eines neuen Geschäftsmodells ist geplant. ${ }^{88}$

\section{eVerlage}

Bei eVerlage handelt es sich um ein Projekt der Universitätsbibliothek Bielefeld, der Staats- und Universitätsbibliothek Göttingen und der THWK Jena in Kooperation mit dem Oldenburger Forschungs- und Entwicklungsinstitut für Informatik-Werkzeuge und Systeme ${ }^{89}$ (OFFIS). Das Projekt eVerlage ist aus den Projekten MeDoc und InterDoc hervorgegangen und ist nach Abschluss der Projektphase nicht weitergeführt worden. Zielsetzung war die Entwicklung einer technischen Infrastruktur, die es kleinen und mittelständischen Verlagen ermöglicht, ihre Produkte (Monographien und Zeitschriften) auf einer elektronischen Plattform zur Verfügung zu stellen. Das Konzept von eVerlage folgte der Idee der „information just in time und just on demand“. Die Kunden der Verlage erwarben nicht das Produkt, vielmehr erwarben sie den Zugriff auf das Produkt. Das Herunterladen des Produktes war nicht erlaubt.

Für die Realisierung dieses Konzeptes hat OFFIS eine technische Infrastruktur entwickelt, die es den Verlagen ermöglichte, ihre Produkte online im Volltext zur Verfügung zu stellen. Ein Schwerpunkt bestand in der Entwicklung eines komplexen Abrechnungs- und Zahlungsverfahrens (Paybox, Kreditkarte, Gutschein, Rechnung). In Anlehnung an verschiedene Nutzergruppen wurde das jeweilige Zahlungsverfahren differenziert (Micropayment). eVerlage unterschied zwischen dem anonymen Gastnutzer, dem registrierten Gastnutzer, Nutzergruppen und dem institutionellen Kunden. Dem anonymen Gastnutzer wurde die Zahlungsart paybox, Geldkarte und Gutschein angeboten, der registrierte Gastnutzer verfügte über ein Konto bei eVerlage, Nutzergruppen und institutionellen Kunden wurde neben den genannten Verfahren die Rechnungsstellung (unter Angabe der vollen Identität) angeboten.

Als ein weiterer Baustein ihres Geschäftsmodells wurden den Kunden Lizenzen angeboten. Hierzu zählen Einzellizenzen (1/2 jähriger Zugriff auf das Medium zu $10 \%$ des Ladenpreises), Gleitlizenzen (dem Medium wird eine bestimmte Anzahl von Zugriffen zugeordnet), Kurzzeitlizenzen (für Einzelnutzer wird ein einstündiger Zugriff auf ein Medium für 50 Cent angeboten) und Campuslizenzen (Zugriff für eine unbegrenzte Anzahl von Nutzern) angeboten.

Die beteiligten Verlage haben sich jedoch von der Bereitstellung der Monographien als elektronischer Ressource distanziert. Elektronische Zeitschriften wurden gar nicht erst verfügbar gemacht. Begründet wurde der Rückzug aus dem Projekt mit fehlenden Ressourcen für die notwendige Digitalisierung der Medien durch die Verlage.
Diese Aufgabe konnte von den kleinen bis sehr kleinen Fachverlagen ${ }^{90}$ nicht sichergestellt werden. Es stellt sich jedoch grundsätzlich die Frage, ob Bibliotheken sich an der Entwicklung von Zahlungsverfahren beteiligen sollten, die den Zugriff auf die Information im elektronischen Umfeld künstlich einschränken.

\section{FIGARO ${ }^{91}$ (German Academic Publishers / Roquade)}

Das Project FIGARO wurde Ende des Jahres 2003 aufgrund fehlender Mittel eingestelt. Bei FIGARO handelte es sich um ein Kooperationsprojekt der niederländischen Universitäten Utrecht und Delft, der deutschen Universitätsbibliotheken Hamburg (Regionales Rechenzentrum) und Oldenburg, dem Universitätsverlag in Florenz und dem Softwarehaus Daidalos in den Niederlanden, die auf europäischer Ebene eine elektronische Publikationsumgebung für Universitätsverlage, kleine, mittelständische Verlage und Fachgesellschaften realisieren wollten ${ }^{92}$. In FIGARO wurde das niederländische Projekt Roquade ${ }^{93}$ und das deutsche Projekt German Academic Publishers (GAP) ${ }^{94}$ integriert, die den Aufbau einer entsprechenden Infrastruktur auf nationaler Ebene verfolgen. Diese Projekte werden weitergeführt Der modulare Aufbau von Figaro ermöglichte die Unterstützung von Verlagen bei der Herausgabe von elektronischen Zeitschriftenpublikationen und Monographien sowie die Publikation von preprints und postprints. Die Projektpartner boten selbst keine verlegerischen Tätigkeiten (Marketing, Vertrieb, Peer-Review) an, sondern arbeiteten an der Entwicklung geeigneter Dokumentmodellierungen und eines www-basierten gemeinsamen workflows, an der Realisierung geeigneter Geschäftsmodelle für das e-publishing und dem Aufbau einer Netzwerkorganisation und einer Plattform für die Entwicklung neuer Technologien und neuer Standards ${ }^{95}$. Die Herausgabe von Zeitschriften auf der Basis des freien Zugangs (open access) war geplant.

${ }^{87} \mathrm{Vgl}$. ausführlicher zu weiteren Bestandteilen der Refinanzierung: Joseph, Heather D.: BioOne: building a sustainable alternative publishing model for non-profit publishers. In: Learned Publishing 16 (2003) S. 134-138.

${ }^{88}$ Joseph (Anm. 87) S. 137.

${ }^{89}$ Zugang: <http://www.offis.uni-oldenburg.de>.

${ }^{90}$ Bei den Verlagen handelt es sich überwiegend um Verlage der Fachgebiete Ingenieurwissenschaften, Naturwissenschaften und den technischen Berufen.

91 Zugang: <http://www.figaro-europe.net/>.

${ }^{92}$ Daneben existieren weitere Kooperationspartner: Universitätsverlag Leuven, Forschungs- und Entwicklungsabteilung NetLab der Lund Universität, der Verlag Lemma in den Niederlanden und DiG in Polen, die Forschungsgruppe Delft Cluster und SPARC USA. Zugang: <http://www. figaroeurope.net/index2.html?mission.html> (15.02.03).

${ }_{93}$ Zugang: <http://www.roquade.nl>.

94 Zugang: <http://www.ubka.uni-karlsruhe.de/gap-c/index_ de.html>

${ }_{95} \mathrm{Vgl}$. Savenije, Bas: Who pays the Ferryman? About new models for scientific communication. Paper presented at the international symposium "Science \& Engineering Libraries for the $21^{\text {st }}$ Century. (2002). Zugang: <http://www.figaro-

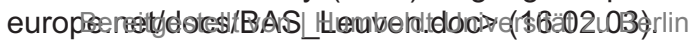




\section{EMS Publishing House ${ }^{96}$}

Der Verlag der Europäischen Mathematischen Fachgesellschaft wurde im Jahr 2002 im Rahmen einer Stiftung in der Schweiz gegründet. Die Aufsicht über den Verlag nimmt der Stiftungsrat wahr, der sich überwiegend aus Mathematikern aus dem Kreis der Europäischen Mathematischen Fachgesellschaft ${ }^{97}$ zusammensetzt. Der Verlag ist rechtlich und finanziell von der Gesellschaft getrennt und nur über den Stiftungsrat verbunden. Die Motivation zur Gründung eines Fachverlages resultierte aus der weitverbreiteten Unzufriedenheit vieler Wissenschaftler mit den kommerziell agierenden Verlagen.

EMS Publishing House verfügt derzeit noch über ein sehr kleines Portfolio an Zeitschriften und Monographien ${ }^{98}$. In der Zukunft ist die Herausgabe weiterer Zeitschriften, Monographien, Lehrbücher, Lecture Notes geplant. Zeitschriften werden als elektronische und als Printversion herausgegeben. Die Printversionen, so der Geschäftsführer Dr. Thomas Hintermann, sind derzeit noch ein klares Muss, jedoch werden diese in ihrer Bedeutung in Zukunft abnehmen ${ }^{99}$.

Der Verlag wendet das traditionelle Geschäftsmodell der Subskription/Lizenzierung der Dokumente an. Als nonprofit-Organisation arbeitet der Verlag jedoch auf der Basis der Kostendeckung und wird seine Produkte zu wissenschaftsfreundlichen Preisen anbieten.

Im Gegensatz zu HighWirePress bestehen zwischen den Fachgesellschaften und dem Verlagshaus jedoch keine verbindlichen Absprachen bezüglich einer Kooperation. Dies zwingt EMS Publishing House dazu, mit den Fachgesellschaften und Instituten Einzelverhandlungen über die Veröffentlichung von Zeitschriften etc. bzw. über andere Kooperationsmodelle zu führen.

\section{Bewertung}

Die beschriebenen Initiativen und Unternehmungen haben einen identischen Ansatzpunkt: Sie unterstützen bzw. streben eine Unterstützung der Fachgesellschaften und kleiner, mittelständischer Verlage bei der Produktion und Distribution ihrer Produkte im Internet an. In vielen Fällen verfügen diese weder über die technischen Kompetenzen noch über die finanziellen Ressourcen, um das Internet als Distributionskanal für ihre Produkte auszunutzen. Dabei ist der Zugriff auf elektronische Volltexte einer Zeitschrift insbesondere in den sich dynamisch entwickelnden STMGebieten immer stärker erforderlich. Fachgesellschaften und Verlage, die den (institutionellen und privaten) Endnutzern diesen Zugriff nicht anbieten können, werden in Zukunft auf dem Markt wissenschaftlicher Information nicht konkurrenzfähig sein.

Durch die neuen Partnerschaften gelingt es den Fachgesellschaften und Verlagen ihren Kundenkreis der potenziellen Abnehmer der wissenschaftlichen Information zu erweitern.

Hinsichtlich ihrer wirtschaftlichen Tragfähigkeit ist das Konzept von HighWirePress, Project MUSE und BioOne als erfolgreich zu bezeichnen. In Anlehnung an das traditionelle Geschäftsmodell der Subskription/Lizenzierung/ pay-per-view (SLP) erbringen diese Unternehmungen ihre Dienstleistungen zu wissenschaftsfreundlichen Preisen, indem sie auf der Basis der Kostendeckung arbeiten. Es muss jedoch kritisch angemerkt werden, dass die beteiligten Fachgesellschaften zur Zeit noch ein geringes Interesse an einem Geschäftsmodell auf der Basis des open access haben. Lediglich BioOne experimentiert bei einigen Zeitschriften mit einem neuen Geschäftsmodell, welches den freien Zugang gewährleistet.

\subsection{Kostensenkung durch direkten Wettbewerb (,Alternative Program“ - SPARC)}

Im Rahmen des „Alternative-Program“ verfolgt SPARC ${ }^{100}$ die Strategie, hochpreisigen, renommierten STM-Zeitschriften direkte Konkurrenzprodukte als „head-to-headcompetitor" gegenüberzustellen. Mit der Herausgabe dieser wesentlich preiswerteren Zeitschriften sollen die jährlichen Preissteigerungen für Zeitschriften kommerzieller Verlage verlangsamt werden. Von den beteiligten Verlagen werden derzeit folgende Zeitschriften in unterschiedlichen Fachgebieten herausgegeben (vgl. Tabelle 8).

Die Zeitschriften sind überwiegend auf der Basis des traditionellen Geschäftsmodells erhältlich. Für den Aufbau einer Kooperation im Rahmen des „Alternative Program“ legt SPARC folgende Kriterien zugrunde:

- Einfluss auf den Markt: Priorität haben solche Projekte, die dazu beitragen, den Wettbewerb auf dem Markt wissenschaftlicher Information zu befördern und die zur Verbreitung wissenschaftlicher Forschungsergebnisse beitragen,

- internationale Perspektive,

- Nutzung der neuen Technologien zur Kostenreduzierung und weitere wettbewerbsfördernde Aktivitäten und Mehrwertdienste,

- klare Zielstellung,

- Reputation der Kooperationspartner und bibliotheksfreundliche Geschäftspraktiken,

96 Zugang: <http://www.ems-ph.org>.

$97<$ http://www.emis.de/ems-general.html>.

98 EMS Publishing House kooperiert mit der amerikanischen Fachgesellschaft AMS auf einer marktwirtschaftlichen Basis mit dem Ziel, das EMS Publishing House als Partner aufzubauen. Zur Zeit stellt die AMS die elektronische Zeitschrift des Verlages EMS auf ihrem Server bereit und bindet diese in den Alert Service ein. (Schriftwechsel mit Dr. Thomas Hintermann am 28.08.2003).

99 Schriftwechsel am 27.08.2003 mit dem Geschäftsführer Dr. Thomas Hintermann.

${ }^{100}$ Weitere Arbeitsschwerpunkte von SPARC sind das Leading Edge Programm und SPARC Scientific Communities. Mit SPARC Leading Edge wird die Entwicklung neuer Modelle wissenschaftlicher Publikation unterstützt. Hierzu zählen die Einführung neuer Technologien bzw. innovative Geschäftsmodelle (z.B. New Journal of Physics, Internet Journal of Physics, Documenta Mathematica, Journal of Insect Science). Mit SPARC Scientific Communities will die Initiative die Kooperation zwischen Wissenschaftlern, Fachgesellschaften und akademischen Institutionen unterstützen. Dies geschieht durch eine offensive Informationspolitik und finanzielle Unterstützung von innovativen Projekten (z.B. eScholarship, Project Euclid, BioOne). Vgl hierzu ausführlicher: Case, Mary M.: Igniting Change in Scholarly Communication: SPARC, Its Past, Present, and Future. In:

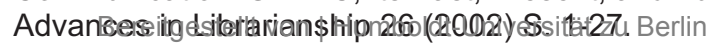




\begin{tabular}{|c|c|}
\hline $\begin{array}{c}\text { Titel/Verlag } \\
\text { kommerzieller Zeitschrift }\end{array}$ & $\begin{array}{c}\text { Zeitschrift des } \\
\text { „Alternative Program“ }\end{array}$ \\
\hline $\begin{array}{l}\text { Tetrahedron Letters } \\
\text { (Reed Elsevier) }\end{array}$ & $\begin{array}{c}\text { Organic Letters } \\
\text { (American Chemical Society) }\end{array}$ \\
\hline $\begin{array}{r}\text { Journal of } \\
(\operatorname{Re} \epsilon\end{array}$ & $\begin{array}{l}\text { Chrystal G } \\
\text { (American }\end{array}$ \\
\hline $\begin{array}{l}\text { Evolutic } \\
\text { (Wolt }\end{array}$ & $\begin{array}{l}\text { Evolutionary Ecology Re- } \\
\text { search } \\
\text { (Michael Rosenzweig) }\end{array}$ \\
\hline $\begin{array}{c}\text { Chemical Physics Letters } \\
\text { (Reed Elsevier) }\end{array}$ & $\begin{array}{c}\text { PhysChemComm } \\
\text { (Royal Society of Chemistry) }\end{array}$ \\
\hline $\begin{array}{l}\text { Organic Geochemistry } \\
\text { (Reed Elsevier) }\end{array}$ & $\begin{array}{l}\text { Geochemical Transactions } \\
\text { (Royal Society of Chemistry) }\end{array}$ \\
\hline $\begin{array}{l}\text { Sensors } \\
\quad(\operatorname{Re}\end{array}$ & IEEE S \\
\hline $\begin{array}{r}\text { Topol } \\
\text { (Reed El }\end{array}$ & $\begin{array}{r}\text { Geometry } \\
\text { (Mathematis } \\
\text { Universität }\end{array}$ \\
\hline $\begin{array}{r}\text { Journal of Los } \\
\text { braic Prog } \\
\text { (Reed E }\end{array}$ & $\begin{array}{l}\text { Theory \& Practice of Logic } \\
\text { Programming (TPLP) } \\
\text { (Cambridge University Press) }\end{array}$ \\
\hline $\begin{array}{c}\text { Topology and Its Applica- } \\
\text { tions } \\
\text { (Reed Elsevier) }\end{array}$ & $\begin{array}{c}\text { Algebraic \& Geometric Topo- } \\
\text { logy } \\
\text { (Institute of Mathematics an } \\
\text { der Universität von Warwick) }\end{array}$ \\
\hline $\begin{array}{l}\text { Mach } \\
\text { (Wolt }\end{array}$ & $\begin{array}{l}\text { Journal of Machine Learning } \\
\text { Research } \\
\text { (JMLR Inc. in Kooperation mit } \\
\text { MIT Press) }\end{array}$ \\
\hline $\begin{array}{c}\text { European Economic Re- } \\
\text { view } \\
\text { (Reed Elsevier) }\end{array}$ & $\begin{array}{l}\text { Journal of the European Eco- } \\
\text { nomic Association }\end{array}$ \\
\hline
\end{tabular}

Tabelle 8: Konkurrenzzeitschriften des „Alternative Program" von SPARC

- die Kooperationspartner verfolgen die Interessen der Bibliothek, der Autoren und Nutzer hinsichtlich des geistigen Eigentums, Nutzungsrechte und Archivierung,

- Herausgebeber und Autoren sichern die Qualität der Zeitschrift,

- langfristige wirtschaftliche Tragfähigkeit der Partner,

- ausreichend vorhandene Kapazitäten für das Management des Projekts und für die Bewältigung der Vereinbarungen ${ }^{101}$.

\section{Neue Ansätze in den Begutachtungsverfahren}

\section{Kritik am traditionellen Begutachtungsverfahren (Peer Review) ${ }^{102}$}

Auch für den Prozess der Begutachtung und Qualitätssicherung eröffnen sich mit dem Einsatz der Informations- und Kommunikationstechnologien neue Möglichkeiten. Eine Auseinandersetzung erscheint vor dem Hintergrund der anhaltenden Kritik an dem Verfahren in diesem Zusammenhang sinnvoll.

Für den wissenschaftlichen Publikationsprozess ist das Begutachtungsverfahren (Peer-Review) der Schlüsselprozess für die Qualitätsischerung wissenschaftlicher
Forschungsergebnisse. Die Veröffentlichung zuvor qualitätsgeprüfter Papiere in Fachzeitschriften ermöglicht den Wissenschaftlern eine Einschätzung über lesenswerte und weniger lesenswerte Artikel. Insofern hat das Begutachtungsverfahren Einfluss darauf, was in der Wissenschaftlergemeinschaft öffentlich diskutiert wird. Darüber hinaus dient auch die Zeitschrift als "Marke" und ihr Markenwert als ein weiterer Qualitätsfilter.

Harnad beschreibt die Bedeutung der Qualitätsprüfung folgendermaßen: „Peer review is a quality-control and certification (QC/C) filter necessitated by the vast scale of learned research today. Without it, no one would know where to start reading in the welter of new work reported every day, nor what was worth reading, and believing, and trying to build one's own further research upon."103 Mit der Durchführung des Begutachtungsprozesses werden in der Regel Wissenschaftler des jeweiligen Fachgebietes betraut. Hierdurch soll die Richtigkeit und Fundiertheit der wissenschaftlichen Arbeit sichergestellt werden, wissenschaftliche Standards gewahrt werden, irrelevante und auch falsche Ergebnisse aus dem wissenschaftlichen Kommunikations- und Publikationsprozess herausgehalten werden. ${ }^{104}$ Die Wissenschaftler erhalten für diese Tätigkeit in der Regel keine Aufwandsentschädigung, jedoch einen Reputationsgewinn, indem sie für eine renommierte Zeitschrift tätig werden.

Für den Begutachtungsprozess hat sich in der Printumgebung das doppelt-blinde Verfahren durchgesetzt, d.h. Autor und Gutachter sind einander nicht bekannt. Von Weller wird der Begutachtungsprozess folgendermassen beschrieben: „Editorial peer review today is a fairly welldefined process. A manuscript is received in an editorial office. The journal editor, or for larger journals one of the associate editors, sends the manuscripts to two or three reviewers. Reviewers are asked to assess the manuscript and make a recommendation to accept, accept with revisions, or reject. The editor or associate editors then decide if they will accept the recommendation of the reviewers. If reviewers disagree, the editor may submit the manuscript to more review or may act as adjudicator and decide if the manuscript should be accepted or rejected." 105

${ }^{101} \mathrm{Vgl} .<\mathrm{http}: / /$ www.arl.org/sparc/core/index.asp?page=c1 $>$.

${ }^{102}$ Vgl. ausführlicher: Peek, Robin P. \& Newsby, Gregory B. (Hg.): Scholarly Publishing: The Electronic Frontier. Cambridge, MA: The MIT Press; Meadows, A. J. 1998: Communicating Research. San Diego, CA: Academic Press; Tenopir, Carol; King, Donald W.: Towards Electronic Journals: Realities for Scientists, Librarians and Publishers. Washington DC, 2000, SLA Publishing. Vgl. auch die Artikel der Peer Review Kongresse auf dem biomedizinischen Gebiet. Zugang: <http://www.ama-assn.org/public/peer/peerhome. $\mathrm{htm}>$ (29.05.03).

${ }^{103}$ Harnad, Stevan: Free at Last: The Future of Peer-Reviewed Journals. In: D-Lib Magazine 5 (1999) 12. Zugang: <http:// www.dlib.org/dlib/december99/12harnad.html> (20.03.03). ${ }^{104}$ Vgl. Meier (Anm. 8) S. 52.

${ }^{105}$ Weller, Ann C.: Emerging models of editorial peer review in the electronic publication environment, 2001. Zugang: <http:// www.icml.org/sunday/publishing/weller.htm> (20.04.03). Vgl. hierzu auch Peters J.: The hundred years war starting today: An exploration of electronic peer review. In: Journal of Electronic Publishing 5 (1996). Zugang: <http://www.press.

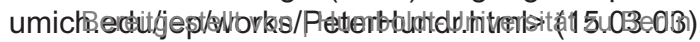


Den Zielsetzungen des Begutachtungsprozesses scheint das derzeitige Verfahren des peer review nicht länger gerecht zu werden. Dieser Eindruck entsteht durch die starke Kritik an dem traditionellen Verfahren ${ }^{106}$. Bemängelt wird die Langsamkeit des Prozesses, die hohen Kosten für die Begutachtung, die Subjektivität der Bewertungen, die mögliche Befangenheit der Gutachter, die Missbrauchsanfälligkeit des gegenwärtigen Systems und das Fehlen von Mechanismen zur Aufdeckung von vorsätzlichen Fehlern, Mängeln und Täuschungen ${ }^{107}$. Im einzelnen bedeutet dies nach Williamson, Mitherausgeber der British Medical Journal Group:

Subjektivität: Vom Herausgeber der Zeitschrift wird das Manuskript abgelehnt, ohne eine Stellungnahme durch einen Gutachter einzuholen bzw. der Herausgeber wählt einen Gutachter aus, der für eine rigorose Ablehnungspraxis bekannt ist.

Befangenheit: Diskriminierung von Autoren aufgrund der Nationalität, der Sprache, des Geschlechts oder der Zugehörigkeit zu einer Forschungsinstitution. Befangenheit kann auch durch ein Konkurrenzverhältnis zwischen Gutachter und Autor auftreten, die in einem gleichartigen Forschungsgebiet tätig sind.

Missbrauchsanfälligkeit: Missbrauch des Systems kann durch Autoren, Professoren und Gutachter gleichermaßen erfolgen. Autoren „verteilen“ ihr Forschungsergebnis auf eine Vielzahl von Artikeln bzw. duplizieren ihre Veröffentlichungen. Als Missbrauch wird jedoch auch die Veröffentlichung von Forschungsergebnissen angesehen, die von wissenschaftlichen Mitarbeitern erarbeitet wurden, jedoch von betreuenden Professoren unter ihrem Namen veröffentlicht werden. Missbrauch liegt auch dann vor, wenn nicht selbst erarbeitete Forschungsergebnisse durch die Gutachter, die mit dem Peer Review betraut wurden, veröffentlicht werden.

In dem gegenwärtigen System ist die Aufdeckung von vorsätzlichen Fehlern, Mängeln und Täuschungen so gut wie unmöglich ${ }^{108}$.

Das doppelt-blinde-Verfahren wird auch als „black-boxVerfahren"109 bezeichnet, da die Gründe für die Ablehnung eines Manuskriptes nicht öffentlich gemacht werden. Dies kann unter Umständen dazu führen, dass innovative Ansätze in einer Disziplin nicht publiziert werden, weil es sich bei den Gutachtern um etablierte Repräsentanten des Faches handelt, die Neuerungen eher skeptisch gegenüberstehen. Aus diesem Grund wird das gegenwärtige Verfahren von Roberts (1999) auch als konservativ und anti-innovatorisch kritisiert ${ }^{110}$.

Agger (1990) macht auf einen weiteren Aspekt aufmerksam: Printversionen von Zeitschriften verfügen in der Regel nicht über ausreichend Seitenkapazität, um eine Vielzahl von Artikeln zu publizieren. Die hohe Ablehnungsquote insbesondere der internationalen und renommierten Zeitschriften wird von inm nicht nur auf den Mangel an fachlicher Qualität der Artikel zurückgeführt, sondern auf die Zwänge des traditionellen Publikationsmodells, nach denen z.B. Zeitschriften eine bestimmte Seitenanzahl nicht überschreiten sollten ${ }^{111}$.

Von Pöschl (2000), Wissenschaftler am Institut für Wasserchemie an der TU München, werden mehrere Gründe für das Versagen des Peer-Review-Verfahrens genannt:

a. „Der hohe Spezialisierungsgrad in den einzelnen Forschungsrichtungen führt dazu, dass selbst Kollegen innerhalb der gleichen Disziplin in vielen Fällen nicht über ausreichendes Detailwissen verfügen, um als Referee [...] eine tiefgehende kritische Betrachtung der eingereichten Manuskripte durchzuführen.

b. In der oft schwer überschaubaren Vielfalt der aktuellen Forschungsaktivitäten, -einrichtungen und -spezialgebiete kann es leicht dazu kommen, dass die im Einzelfall bestqualifizierten Referees den Editoren nicht bekannt sind.

c. Auch Wissenschaftler, die als Referees bestens qualifiziert und den Editoren bekannt sind, können häufig nicht genügend Zeit für einen gründlichen Review aller innen zugeteilten Manuskripte aufwenden.

d. Schließlich sind manche Referees aufgrund von Konkurrenzsituationen eher an einer Verlangsamung/ Behinderung der Publikationen ihrer Konkurrenten interessiert als an einer konstruktiven Kritik und Verbesserung der von innen begutachteten Manuskripte.."112

Die Diskussion um die Reformierung des traditionellen Begutachtungsverfahrens im elektronischen Umfeld ist insbesondere durch Stevan Harnad vorangetrieben worden. In seinen Aufsätzen setzt er sich mit dem Innovationspotenzial der Internettechnologien für das Begutachtungsverfahren auseinander ${ }^{113}$. Die Vorteile, die sich für den Begutachtungsprozess durch das elektronische Publizieren und die Verbreitung durch das Internet erge-

${ }^{106}$ Vgl. hierzu auch Ginsparg, Paul: Winners and Losers in the Global Research Village. Unesco Expert Conference on Electronic Publishing, Paris 1996. Zugang: <http://arxiv. org/blurb/pg96unesco.html> (18.03.03).

${ }^{107}$ Williamson, Alex: What Happens to Peer Review? Papier anlässlich des ALPSP-International Learned Journals Seminar, London 2002. Zugang: <http://www.alpsp/org/wil120402. ppt> (15.05.03).

${ }^{108}$ Vgl. Rowland, Fytton: The Peer Review Process. Report to the JISC Scholarly Communication Group.(ohne Datum). Zugang: <http://www.jisc.ac.uk/uploaded_documents/ rowland.pdf> (03.04.03).

${ }^{109}$ Peters, John: The hundred years war starting today. An exploration of electronic peer review Journal of electronic Publishing 5 <http://www.press.umich.edu/jep/works/PeterHundr.html>.

${ }^{110}$ Roberts, Peter: Scholarly Publishing, Peer-Review and the Internet. In: FirstMonday 4 (1999). Zugang: <http:// www.firstmonday.dk/issues/issue4 4/proberts/index.html> (02.04.03).

${ }^{111}$ Vgl. Agger, Ben : The Decline of Discourse: Reading, Writing and Resistance in Postmodern Capitalism. London 1990.

${ }^{112}$ Pöschl, Ulrich: Interaktive wissenschaftliche Fachzeitschriften. Die Zukunft der wissenschaftlichen Publikation und Diskussion in elektronischen Medien. Unveröffentlichtes Papier. München 2000. S. 1; vgl. hierzu auch <http://www.nature. com/cgi-taf/DynaPage.taf?file=/nature/journal/v416/n6878/ full/416258a_r.html> (Zugang 14.03.03).

${ }^{113}$ Harnad, Stevan: Implementing peer review on the Net: Scientific quality control in scholarly electronic journals. In: Peek, Robin P. \& Newby, Gregory (Hg.): Scholarly Publishing: The Electronic Frontier. Cambridge, Mass. MIT Press, 1996. S. 112; Harnad, Stevan: Learned Inquiry and the Net: The Role of Peer Review, Peer Commentary and Copyright, 1998. Zugang: <http://www.ecs.soton.ac.uk/ harnad/Papers/Har-

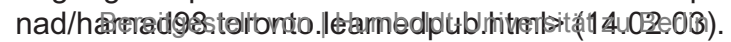


ben, beschreibt er folgendermaßen: "[...] the Net does offer the possibility of distributing the burdens of peer review more equitably, selecting referees on a broader and more systematic basis (electronic surveys of the literature, citation analysis, even posting calls for reviewers to pertinent professional experts' bulletin boards and allowing those who happen to have the time to volunteer themselves). The speed, with which a manuscript can be circulated electronically is also an advantage, as is the convenience that many are discovering in reading and commenting on manuscripts exclusively on-screen."114

Die Vorteile eines elektronischen Peer Review liegen nach Harnad also vor allem in der Beschleunigung des Begutachtungsprozesses (und daraus resultierend einer schnelleren Publikation der Ergebnisse) und der Beteiligung einer größeren Anzahl von Gutachtern am Begutachtungsprozess ${ }^{115}$, wodurch sich eine größere Transparenz erzielen lässt.

\subsection{Konzept des Peer-Commentary - Beispiele}

Zu Beginn der 90er Jahre ist von Stevan Harnad das Konzept des „Open Peer Commentary“ entwickelt worden ${ }^{116}$. Als Herausgeber der Zeitschriften „Behavioral and Brain Sciences“ und der Zeitschrift „Psycoloquy“ verfügt er über zahlreiche Erfahrungen mit der Durchführung von Begutachtungsprozessen. Der Kognitionswissenschaftler sieht das Konzept des „Open peer commentary“ nicht als Ersatz für den traditionellen Begutachtungsprozess an, sondern als Ergänzung bzw. als einen zweiten Begutachtungsschritt.

Im traditionellen Begutachtungsprozess findet die Beurteilung durch eine ausgewählte Gruppe von Gutachtern anonym und vor der Publikation statt. Das Manuskript wird auf seine Publikationswürdigkeit hin bewertet.

Bei dem Konzept des „open peer commentary“ wird dieser Begutachtungsprozess durch eine strukturierte, offene Diskussion zwischen Gutachtern, Autoren und anderen Interessierten in einem elektronischen Forum ergänzt. Der Verlauf des Gesprächs über das Manuskript ist in einem elektronischen Archiv abgebildet und steht Interessierten zur Verfügung. Der Prozess der offenen online-gestützten Fachdiskussion wird von Harnad auch als „scholarly skywriting" bezeichnet. Die Interaktion der beteiligten Akteure, die Transparenz und die Beschleunigung des Verfahrens sieht Harnad als die eigentlich "revolutionäre Dimension" des wissenschaftlichen Kommunikationsprozesses an ${ }^{117}$. Das Konzept des peer commentary kommt in verschiedenen Zeitschriften zur Anwendung.

\section{Psycoloquy}

Die seit 1989 erscheinende Zeitschrift Psycoloquy ist eine internationale, interdisziplinäre, elektronische Zeitschrift, die von der American Psychological Association (APA) finanziert wird und die Fachgebiete Kognitions- und Verhaltenswissenschaften, Linguistik, Psychologie und Philosophie umfasst. Die Zeitschrift strebt eine intensive und nichtanonymisierte Interaktion zwischen den Autoren, Gutachtern und anderen Interessierten an. Mit dem Einreichen des Manuskriptes muss der Autor begründen, weshalb eine öffentliche Diskussion zu dem Themengebiet notwendig ist und welche Interessen sich mit der öffent- lichen Begutachtung verbinden. Die Gutachter erwarten eine schlüssige Argumentation und die Fähigkeit des Autors, sich auf die Vorgaben der Gutachter zu beziehen und diese zu strukturieren. Wird das Manuskript in einem ersten Schritt als publikationswürdig angesehen, wird es zwischen Gutachtern, Autoren und Interessierten in dem Online-Forum diskutiert. Nach dem Begutachtungsverfahren wird nicht nur das Manuskript, sondern auch der Diskussionsverlauf elektronisch verfügbar gemacht.

\section{Journal of Interactive Media in Education}

Das seit 1996 von der Open University in Großbritannien herausgegebene Journal of Interactive Media in Education hat einen Begutachtungsprozess eingeführt, der sich an folgenden Grundsätzen orientiert:

- "authors have the right to reply,

- reviewers are named and accountable for their comments, and their contribution acknowledged,

- the wider research community has the chance to shape a submission before publication." 118

Der Begutachtungsprozess dieser Zeitschrift unterteilt sich in die Phasen private open peer review, public open peer review, publication:

1. Private Open Peer Review

Die eingereichten Manuskripte werden an bis zu drei, den Autoren bekannte Gutachter mit der Bitte um eine Stellungnahme und Empfehlung geschickt. Die Kommentare werden von den Gutachtern auf einer nicht-öffentlichen Seite „veröffentlicht“. Obwohl den Gutachtern die Möglichkeit der Anonymität eingeräumt wird, findet der erste Begutachtungsprozess in der Regel nicht anonym statt. Die Autoren sind aufgefordert, zu den eingereichten Kommentaren der Gutachter Stellung zu beziehen.

2. Public Open Peer Review

Auf der Basis der stattgefundenen Diskussion wird das Manuskript als preprint für den öffentlichen Begutachtungsprozess an die relevanten Wissenschaftlergemeinschaften zur Diskussion weitergereicht. Die vorangegangene

${ }^{114}$ Harnad, S.: Scholarly Skywriting and the Prepublication Continuum of Scientific Inquiry. Psychological Science 1 (1990) S. $342-343$

${ }^{115}$ Die Studie von Black, Evans, Godlee, van Rooyen und Smith beschäftigt sich mit den Auswirkungen auf die Begutachtungsergebnisse bei open peer review und blind peer review. Vgl. hierzu ausführlicher: Black, Nick; Evans, Stephen; Godlee, Fiona; van Rooyen, Susan und Richard Smith: Effect of open peer review on quality of reviews and on reviewers' recommendations: a randomised trial. In: British Medical Journal 318 (1999) 71 75, S. 23-27.

${ }^{116} \mathrm{Vgl}$. Harnad, Stevan: The invisible hand of peer review. In: nature web matters, 1998. Zugang: <http://www.nature.com/ nature/webmatters/invisible/invisible.html> (20.03.03); vgl. hierzu auch: Harnad, Stevan: Creative Disagreement. Open peer Commentary adds a vital dimension to review procedures (Preprint o. D.). Zugang: <http://www.lancs.ac.uk/ug/ wangx1/extra/test_file/testfile17.htm> (15.03.03).

117 Vgl. Harnad, Stevan: Implementing Peer Review on the Net: Scientific Quality Control in Scholarly Electronic Journals. 1996. Zugang: <http://www.ecs.soton.ac.uk/ harnad/ Papers/Harnad/harnad96.peer .review.html> (09.03.03).

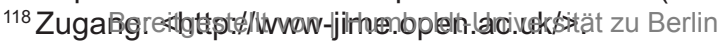


Diskussion bildet den Ausgangspunkt für die in dem erweiterten Forum stattfindende Debatte. Der Prozess der öffentlichen Begutachtung umfasst einen Zeitraum von vier Wochen

Nach Abschluss der Diskussion werden von den Gutachtern die wichtigsten Aspekte in einem Bericht zusammengefasst und als Änderungsvorschläge an die Autoren zurückgegeben.

3. Publication

Während in den konventionellen Zeitschriften die Auseinandersetzung mit der Veröffentlichung des Artikels in der Zeitschrift beginnt, wird die Diskussion bei dieser neuen Form der Begutachtung zu einem früheren Zeitpunkt angesetzt. Neben dem publizierten Artikel werden auch die wichtigsten Kommentare bzw. Diskussionen in die Publikation aufgenommen. Die Publikation ist auf den Seiten der elektronischen Zeitschrift frei zugänglich.

\section{Atmospheric Chemistry and Physics ${ }^{119}$}

Atmospheric Chemistry and Physics (ACP) ist eine elektronische Zeitschrift, die von der Europäischen Geophysikalischen Gesellschaft publiziert wird. Die Herausgeber dieser Zeitschrift sind Wissenschaftler auf dem Gebiet der Atmosphärenforschung.

Der Begutachtungsprozess in dieser elektronischen Zeitschrift ist zweistufig und mit einer öffentlichen Diskussion im elektronischen Raum verbunden. Manuskripte, die als grundsätzlich publikationswürdig eingestuft werden, werden sofort in einem internetbasierten Diskussionsforum der ACP (Atmospheric Chemistry and Physics Discussion -ACPD) veröffentlicht und sind dort für mehrere Wochen zur Diskussion freigegeben. Die Kommentare der Gutachter (anonym oder namentlich) und die Antworten der Autoren sind einsehbar. Nach einem Zeitraum von mehreren Wochen werden die Manuskripte auf der Grundlage dieser öffentlichen Diskussion überarbeitet und anschließend in der Zeitschrift veröffentlicht. Das Manuskript selbst, der Begutachtungsprozess und der Artikel in seiner Endfassung sind mit Seitenzahlen versehen, zitierfähig und frei zugänglich archiviert ${ }^{120}$.

In einer Graphik werden die Begutachtungsprozesse und die dafür vorgesehenen Zeiträume dargestellt' ${ }^{121}(\mathrm{vgl}$. Graphik 9).

\section{Trennung von Veröffentlichung und Begutachtung in elektronischen Archiven - das Konzept der „Overlay Journals“}

Für die Entwicklung eines neuen Publikationsmodells im elektronischen Umfeld wird die Trennung von Begutachtung und Veröffentlichung als wesentlich angesehen ${ }^{122}$. Auf der Basis der in den Repositorien vorhandenen Manuskripte lassen sich die beschriebenen Begutachtungsverfahren (offen, einfach-blind, doppelt-blind) zur Anwendung bringen. In den Empfehlungen des ersten Workshops der Open Archive Initiative $(2001)^{123}$ wird vorgeschlagen, dass die Autoren bzw. die Forschungseinrichtungen, denen diese Autoren angehören, die Kosten für die Begutachtung der Manuskripte in den elektronischen Archiven zu tragen haben. Die begutachteten Manuskripte können in sogenannten
,Overlay Journals' publiziert werden. Hierbei handelt es sich um virtuelle Zeitschriften, die auf der Basis verlinkter Archive herausgegeben werden. Smith beschreibt die vielfältigen Publikationsmöglichkeiten folgendermaßen: „While some of the content for overlay journals might have been previously published in refereed journals, other research may have only existed as a pre-print or work in progress. As a paper could appear in more than one journal and be evaluated by more than one refereeing body, these overlays would allow the aggregation and combination of research articles

- by multiple logical approaches-for example, on a particular theme or topic (becoming the functional equivalent of anthology volumes in the humanities and social sciences

- across disciplines, or

- by affiliation (faculty departmental bulletins that aggregate the research of their members)." "124

Erfahrungen in der Herausgabe von ,Overlay Journals' für preprints liegen bei der American Physical Society (APS) vor, die die Zeitschrift Physical Review herausgibt. Diese ist seit 1996 auch online zugänglich. Die in den Artikeln zitierten Arbeiten verweisen nicht nur auf andere Artikel der Zeitschrift, sondern auch auf Artikel in preprint-Archiven, die in ihrer Anzahl ständig zunehmen. Als Reaktion auf diese Entwicklungen entschied sich die Gesellschaft für die Herausgabe eines, Overlay Journals'. Smith unterscheidet zwei Formen:

- „[...] an overlay on a system of electronic preprints through citations,

- the articles themselves are available and linked as preprints." 125

Die Fachgesellschaft gibt ein ,Pre-Journal' heraus, welches als ,Overlay' die preprints enthält und vor der Herausgabe der qualitätsgeprüften Zeitschriften für die Wissenschaftler bereitgestellt wird. Wird das Manuskript als qualitätsgeprüfter Artikel veröffentlicht, wird der Link auf das preprint entfernt. Smith betont, dass das ,Pre-Journal' sich nicht zu einem Konkurrenten der etablierten Zeitschriften entwickelt hat und nicht zu finanziellen Einbußen führte. „Clearly authors (and readers?) are valuing the journal for more than the simple communication function that the preprints do so well." 126 Um die preprints in den wissenschaftlichen Publikationsprozess einzubeziehen, schlägt die OAI den

119 Weitere Beispiele für öffentliche Begutachtungsverfahren: British Medical Journal, Medical Journal of Australia.

${ }^{120}$ Vgl. Pöschl (Anm. 112) S. 2 ff.

${ }^{121}$ Zugang: <http://www.copernicus.org/EGU/acp/flow_chart1. $\mathrm{htm}>(15.05 .03)$

${ }^{122}$ Vgl. Crow (Anm. 6).

${ }^{123}$ Vgl. hierzu: European Science Foundation: Open access. Restoring scientific communication to its rightful owners, 2003. Zugang. <http://www.arl.org/sparc/SPB21_OAI.pdf> (25.04.03).

${ }^{124}$ Smith, Arthur P.: Overlay Journal. (2002). Zugang: <http:// www2.iro.umontreal.ca/ paquetse/cgi-bin?Overlay_Journal> (16.05.03); vgl. hierzu auch: Smith, Arthur P.: The Journal as an Overlay on Preprint Databases. In: Learned Publishing 13 (2000) Nr. 1, S. 43-48. Zugang: <http://ridge.aps. org/APSMITH/ALPSP/talk1.html> (16.05.03).

${ }^{125} \mathrm{vgl}$. Smith (Anm. 124).

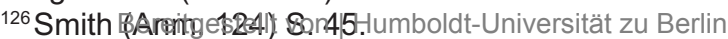


Atmospheric Chemistry and Physics (ACP)

Process of Peer-Review, Publication and Interactive Discussion

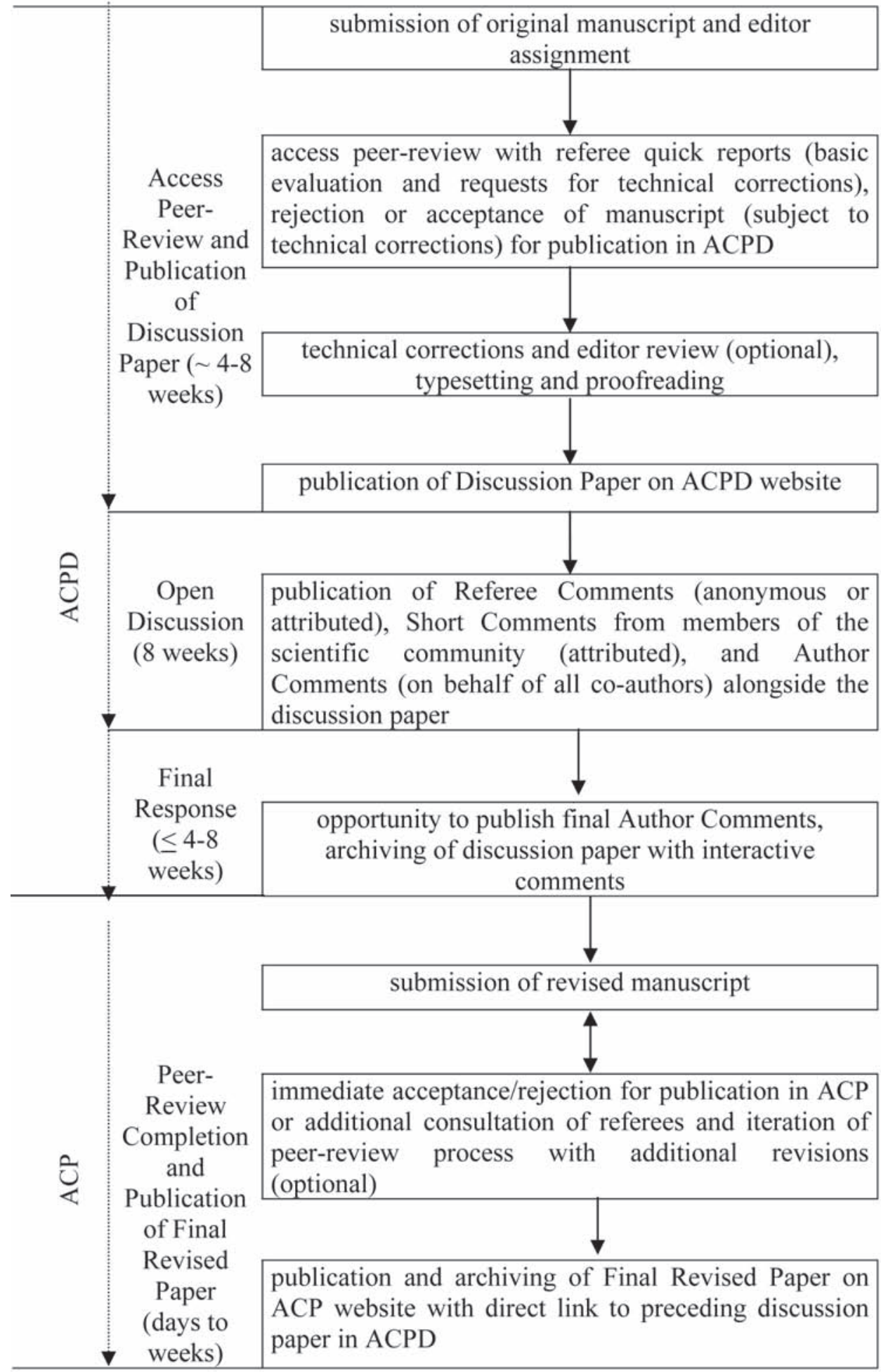

$\longrightarrow \begin{aligned} & \text { continuation of scientific discussion by longer or later } \\ & \text { comments and replies (Peer-Reviewed Comments and } \\ & \text { Replies) which are processed as separate discussion } \\ & \text { papers in ACPD and can achieve publication in ACP }\end{aligned}$


Aufbau mehrerer Archive vor, in denen Dokumente mit einem unterschiedlichen Begutachtungsstatus bzw. Bearbeitungsstatus durch den Autor abrufbar werden.

Paul Ginsparg beschreibt einen zweistufigen Begutachtungsprozess, der auch der Zunahme wissenschaftlicher Publikationen stärker als bislang Rechnung trägt. Während in dem traditionellen Begutachtungsverfahren die Ablehnungsquoten ein Qualitätskriterium für das Renommee der Zeitschrift darstellt, strebt das Modell von Ginsparg eine möglichst umfängliche Veröffentlichung der wissenschaftlichen Information an.

In dem Modell wird zwischen Archiven von grundlegendem Standard (standard tier system) und Archiven mit einem höheren Standard (upper tier system) der wissenschaftlichen Information unterschieden. In das Archiv mit grundlegendem Standard werden solche Manuskripte aufgenommen, die definierten Minimalkriterien entsprechen, d.h., „on a first pass only some cursory examination or other pro forma certification is given for acceptance into a standard tier: this could be minimally labor-intensive, perhaps relying primarily on an automated check of author institutional affiliation, prior publication record, research grant status, or other related background; and involve human labor primarily to adjudicate incomplete or ambiguous results of an automated pass"127.

Von entscheidender Bedeutung ist, dass die Manuskripte eine prinzipielle Publikationswürdigkeit darstellen. „These mechanisms are an important - if not essential - component of why readers find the site so useful: though the most recently submitted articles have not yet necessarily undergone formal review, the vast majority of the articles can, would, or do eventually satisfy editorial requirements somewhere. Virtually all are in that grey area of decidability, and virtually not are entirely useless [...]." ${ }^{\prime \prime 28}$

In den Archiven höheren Standards können sämtliche Verfahren des Peer Review (offenes, blindes, doppelt-blindes Verfahren, peer commentary) zur Anwendung kommen. Die Kriterien für die Begutachtung „[...] could be any of variety of impact measures, to be determined, and based explicitly on their prior widespread and systematic availability and citability: e.g. reader nomination or rating, citation impact, usage statistics, editorial selection [...]. The instructions to expert reviewers would be similar to those now, based on quality, originality, and significance of research, degree of pedagogy (for review articles) [...]."129

Ginsparg sieht den Vorteil eines solchen Systems in der Auswahl hochwertiger Aufsätze, ohne die Verbreitung von Manuskripten und die Beförderung der wissenschaftlichen Kommunikation in der jeweiligen Disziplin einzuschränken.

\section{$4 \quad$ Zusammenfassung}

Preissteigerungen, Konzentrationsprozesse im Verlagswesen, fehlende Geschäftsmodelle, die die Möglichkeiten der neuen Technologien ausschöpfen, sind Ausdruck einer strukturellen Krise des wissenschaftlichen Publikationswesens. Der Konflikt zwischen privater Verwertung und öffentlicher Nutzung verschärft sich mit dem Übergang zum digitalen Medium und verdeutlicht die dominante Rolle der Verlage für die Registrierung, Zertifizierung und Verbreitung der wissenschaftlichen Information sowie die Abhängigkeit der Hochschulen bzw. ihrer Infrastruktureinrichtungen beim Bezug wissenschaftlicher Information.

Die neuen Technologien bieten jedoch die Möglichkeit, den Publikationsprozess im elektronischen Umfeld neu zu gestalten. Gegenwärtig lassen sich vier Aktionsfelder auf dem Gebiet des elektronischen Publizierens beobachten, die auf eine Reformierung des wissenschaftlichen Publikationswesens abzielen: die Herstellung eines kostenlosen Zugangs zur wissenschaftlichen Information auf der Basis neuer Geschäftsmodelle, Produktion und Distribution der wissenschaftlichen Information durch die Hochschulen (Selbstorganisation der Wissenschaften), neue Kooperationsformen zwischen den Akteuren in der Wertschöpfungskette und die Erzielung von Kostensenkungen für wissenschaftliche Information durch eine Verstärkung des Wettbewerbs.

Während die gegenwärtigen Geschäftsmodelle auf der Basis der Subskription, der Lizenzierung und des payper-view (S/L/P) beruhen und den Abnehmer der wissenschaftlichen Information finanziell belasten, werden in dem neuen Geschäftsmodell Artikelbearbeitungsgebühren bzw. Autorengebühren erhoben. Der Zugang zur Information ist in diesem Modell kostenlos und die wissenschaftliche Information erreicht ihre maximale Sichtbarkeit. Die künstliche Beschränkung des Zugangs zur Information erscheint vor dem Hintergrund der Durchsetzung der neuen Technologien zunehmend anachronistisch.

Hochschulen sind im Begriff, elektronische Archive auf fachlicher und institutioneller Ebene aufzubauen. Die Repositorien sind über das OAI-Protokoll recherchierbar, d.h. Ergebnisse aus Lehre und Forschung einer Hochschule bzw. eines Fachgebietes sind weltweit verfügbar. Repositorien bieten ein großes Potenzial zur Reformierung des wissenschaftlichen Publikationswesens, wobei die Entwicklungen auf diesem Gebiet in den verschiedenen Fachgebieten sehr unterschiedlich sind. Die elektronischen Archive bilden die Basis für den Aufbau eines neuen Publikationsmodells, in dem die Hochschulen eine wichtige Rolle als Produzent und Distributor wissenschaftlicher Information einnehmen können. Die Entwicklung geeigneter Begutachtungsverfahren ist in diesem Zusammenhang von großer Bedeutung.

Die Durchsetzung eines neuen Publikationsmodells hängt jedoch entscheidend von der Innovationsbereitschaft der Hochschulen ab. Dazu gehört auch, dass die Wissenschaftler von der Hochschule verstärkt dazu angehalten werden, die beschriebenen Vertragsbedingungen nicht länger zu akzeptieren. Eine Veröffentlichung der wissenschaftlichen Ergebnisse als preprint oder als postprint auf dem Server der Hochschule ist grundlegend für die Entwicklung der elektronischen Archive.

${ }^{127}$ Ginsparg, Paul: Can Peer Review be better Focused, (Preprint) 2003. Zugang: <http://arxiv.org/blurb/pg02pr.html> (22.05.03).

${ }^{128}$ Ginsparg (Anm. 127) S. 4.

${ }^{129}$ Ginsparge(iAvemelit2z) \$H5mboldt-Universität zu Berlin 
Fachgesellschaften, kleine und mittelständische Verlage kooperieren mit öffentlichen und privatwirtschaftlichen Einrichtungen, um ihre Zeitschriften online vertreiben zu können. Im Vordergrund dieser neuen Kooperationsmodelle steht jedoch nicht der freie Zugang zur Information, sondern die Durchsetzung wissenschaftsfreundlicher Preisgestaltungen. In der gegenwärtigen Transformationsphase ist die Anwendung des traditionellen Geschäftsmodells noch erfolgreich.

Die Herstellung von Wettbewerbsbedingungen zwischen den Anbietern wissenschaftlicher Information ist das vierte Aktionsfeld, in dem SPARC im Rahmen seines Alternative Program tätig ist. Durch die Herausgabe preisgünstiger Konkurrenzzeitschriften wird eine Verlangsamung der
Preissteigerung für die Produkte der großen kommerziellen Verlage angestrebt. Gegenwärtig dominieren auch hier die traditionellen Geschäftsmodelle.

\section{Anschrift der Autoren:}

Heike Andermann

Universitätsbibliothek Potsdam

Universitätskomplex III, Haus 5

August-Bebel-Str. 89

D-14482 Potsdam

Dr. Andreas Degkwitz

Universitätsbibliothek Potsdam

Am Neuen Palais 10, Haus 11

D-14469 Potsdam

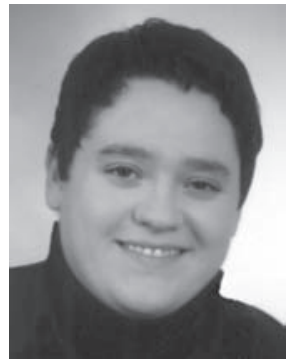

